\title{
Using Diversities to Model the Reliability of N-version Machine Learning System
}

This paper was downloaded from TechRxiv (https://www.techrxiv.org).

\section{LICENSE}

CC BY 4.0

SUBMISSION DATE / POSTED DATE

25-08-2021 / 27-08-2021

CITATION

Machida, Fumio (2021): Using Diversities to Model the Reliability of N-version Machine Learning System. TechRxiv. Preprint. https://doi.org/10.36227/techrxiv.16435656.v1

DOI

10.36227/techrxiv.16435656.v1 


\title{
Using Diversities to Model the Reliability of $\mathrm{N}$-version Machine Learning System
}

\author{
Fumio Machida, Senior Member, IEEE
}

\begin{abstract}
N-version machine learning system (MLS) is an architectural approach to reduce error outputs from a system by redundant configuration using multiple machine learning (ML) modules. Improved system reliability achieved by N-version MLS inherently depends on how diverse ML models are employed and how diverse input data sets are given. However, neither error input spaces of individual ML models nor input data distributions are obtainable in practice, which is a fundamental barrier to understanding the reliability gain by $\mathrm{N}$-version architecture. In this paper, we introduce two diversity measures quantifying the similarities of ML models' capabilities and the interdependence of input data sets, respectively. The defined measures are used to formulate the reliability of an elemental N-version MLS called dependent double-modules double-inputs MLS. The system is assumed to fail when two ML modules output errors simultaneously for the same classification task. The reliabilities of different architecture options for this MLS are comprehensively analyzed through a compact matrix representation form of the proposed reliability model. Except for limiting cases, we observe that the architecture exploiting two diversities tends to achieve preferable reliability under reasonable assumptions. Intuitive relations between diversity parameters and architecture reliabilities are also demonstrated through numerical experiments with hypothetical settings.
\end{abstract}

Index Terms - Diversity, Machine learning system, Redundant architecture, Reliability, Software fault-tolerance

\section{INTRODUCTION}

Q UALITY assurance of machine learning systems (MLSs) is becoming a major concern of system providers who adopt advanced machine learning (ML) functions in their products or services. In the development of MLSs, the desirable input-output relations are not fully specified in advance since the behavior of ML functions is determined by the samples used in the ML training process [21]. Correct outputs are not always guaranteed in real user environments, even if the trained ML functions achieve high accuracy for the testing data set. Therefore, understanding the reliability consequence of error outputs from an ML function is crucial in MLS design, particularly for safety-critical domains such as autonomous vehicles [1].

The N-version machine learning system is an approach to improving the reliability of system outputs by introducing redundant architecture [2]. The idea is analogous to the traditional software fault-tolerant technique referred to as N-

This work was supported in part by a grant of JSPS KAKENHI Grant Number 19K24337.

F. Machida is with the Department of Computer Science, University of Tsukuba, Tsukuba, Japan, (e-mail: machida@cs.tsukuba.ac.jp). version programming that employs $N \geq 2$ functionallyequivalent independent programs from the same initial specification [3][4]. An MLS may employ $\mathrm{N}$ different ML models that are trained independently for the same task. Since individual ML models output errors differently, the probability of simultaneous errors from the N-version MLS can be reduced. To make an N-version MLS effective, it is essential to include the diverse versions of ML models. If all ML models output errors upon the same input data, there is no reliability gain at all. However, it is known that ML models trained by different algorithms or configurations output errors differently for the same input data set [5]. Moreover, outputs from a single ML model can also be diversified by perturbating input data for inference [6][18]. We can obtain diverse outputs not only from different ML models but also from a single ML model by changing input data for inference. Although both the diversity among ML models and the diversity among input data sets significantly affect the reliability of the system output, the model to analyze the reliability gain achieved by the diversities is still underexplored [2].

In this paper, we present an analytical model for characterizing the reliability of output from a basic N-version MLS composed of two ML modules for classification tasks and two sensors generating different input data. An ML module installs an ML model to classify the input data and is connected to either one of the sensors. The MLS outputs classification results when two ML modules agree with the output. We assume that the errors of two ML models are largely similar, and the probability distributions of two input data are not independent of each other. A representative example of such a system is a perception system in an autonomous vehicle equipped with two image sensors and two image classifiers. We call this type of system a dependent double-modules doubleinputs $M L S$ whose applications are not limited to autonomous driving. We believe a dependent double-modules double-inputs MLS becomes a fundamental building block of any larger Nversion MLSs. Modeling the architecture reliability of such a system is an essential step toward designing effective $\mathrm{N}$-version MLS.

Our previous study presented a preliminary reliability model for a dependent double-modules double-inputs MLS [2]. In this paper, we extend our problem scope to a more general case and show general properties that may guide us to choose a preferable architecture for given ML modules and input data sets. First, we formulate the reliability of a dependent doublemodules double-inputs MLS for dependent input data distributions and two error input spaces given by individual ML 
models. Depending on the choice of input data and ML model for individual ML modules, there can be six architecture options (shown in Figure 2 in Section II-C). The choice of architecture affects the reliability of system outputs because error outputs from individual ML modules are not independent. To characterize the reliability difference of six architectures, we introduce two diversity measures, conjunction of errors and intersection of errors, representing the similarity of input data from two sensors and the similarity of error input spaces for two ML models. The diversity measures are used to formulate the architecture reliabilities. The presented reliability model is subsequently used to show some important properties on the reliabilities of different architectures under specific assumptions on the relation between input data distribution and error input spaces. Finally, we conduct numerical experiments to investigate the reliability of a dependent double-modules double-inputs MLS in a hypothetical setting. We use a bivariate normal distribution for input data distribution and define the error input spaces by the closed intervals on a real number. Despite the imaginary setting, the numerical results give an intuitive view of the architecture reliability analysis and demonstrate the properties derived from the proposed model.

The contributions are summarized as follows.

1. We propose a new reliability model for a basic N-version MLS by defining two kinds of diversity measures that characterize the dependencies of two input data distributions and the two error input spaces attributed by corresponding ML models. Our formulation with a compact matrix representation gives a useful tool to investigate the properties of architectures' reliability in relation to the diversity measures.

2. By restricting the type of input data distributions on error input spaces, we show some important properties that can guide the choice of the preferable architecture in terms of system output reliability. Except for limiting cases, our analysis implies that the architecture exploiting two diversities tends to achieve preferable reliability to the architectures relying on a single diversity.

3 . We provide the results of numerical experiments that help understand the dependencies between two input data and two ML models for computing the reliability of a dependent double-modules double-inputs MLS. The experimental results confirm the properties derived from the proposed model.

The rest of the paper is organized as follows. Section II introduces a motivating example and specifies the problem scope; the reliability analysis of a dependent double-modules double-inputs MLS. To compare six possible architecture options, in Section III, we present the diversity measures and use them to formulate the reliabilities of MLS outputs. Section IV shows some important properties we can derive from the constructed reliability models. Section V shows the results of numerical experiments to show the difference of reliabilities achieved by our redundant configuration scheme. Section VI discusses related work and, finally, Section VII gives conclusions and potential future studies.

\section{PRELIMINARIES}

In this section, we first show a motivating example and present the reliability design issue. Then we define our problem scope and introduce the notations used throughout the paper.

\section{A. A motivating example}

As an example of MLS applications, we consider an autonomous vehicle equipped with image sensors and ML modules to classify input images. For safe autonomous driving on the road, the system is required to recognize the traffic signs, signals, other vehicles, and other obstacles using the equipped sensors. Relying on a single sensor and a single ML function is not encouraged since the output of an ML module is highly error-prone to the samples from the real world [7][8]. Thus, Nversion MLS can be adopted for improving the reliability of system output. Figure 1 shows a scenario in which an autonomous vehicle needs to recognize the traffic signal in front of the car using two cameras and two ML modules.

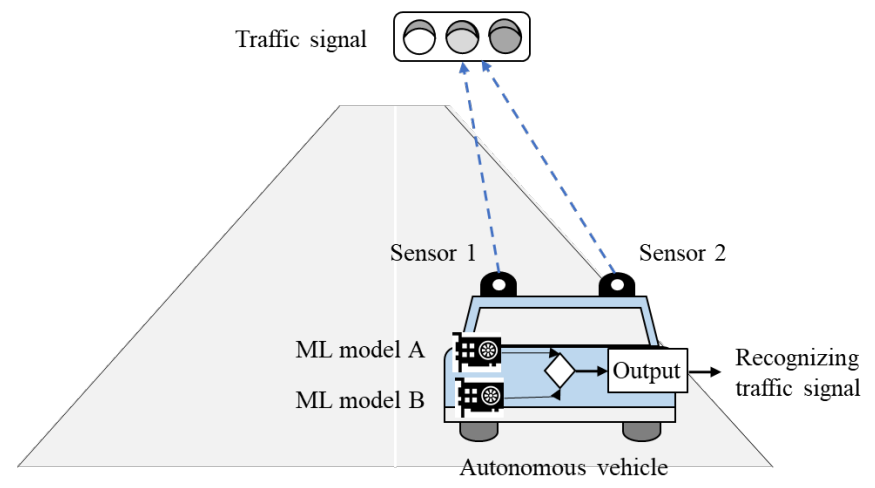

Figure 1. An example scenario for an autonomous vehicle on the road

The cameras can generate different images from slightly different angles. The ML models can behave differently on the same input image by using different ML algorithms or training data sets. When the outputs of these ML modules disagree, the system does not output any inference results to avoid errors. In other words, the system outputs error only when both modules agree on the inference result, but the answer is not correct.

The design issue we encounter in configuring such a dependent double-modules double-inputs MLS is the combination of input data and ML models for individual modules. A wrong combination of input data and ML model even may decrease the system output reliability. In order to discuss this design issue and clarify our problem scope, we introduce formal notations in the following section.

\section{B. Reliability of $M L S$}

We define the reliability of MLS as the probability that an MLS output disagrees with the ground truth in the real world (e.g., red signal). In this paper, we focus on an MLS using MLbased classifiers whose outputs are class labels for the input data. Unlike the accuracy measures of classification tasks, we do not distinguish false positives from false negatives. Errors can also be caused by the implementation bugs of ML programs [9][15]. Any outputs that do not match the ground truth are considered as errors which causing unreliable system outputs. 


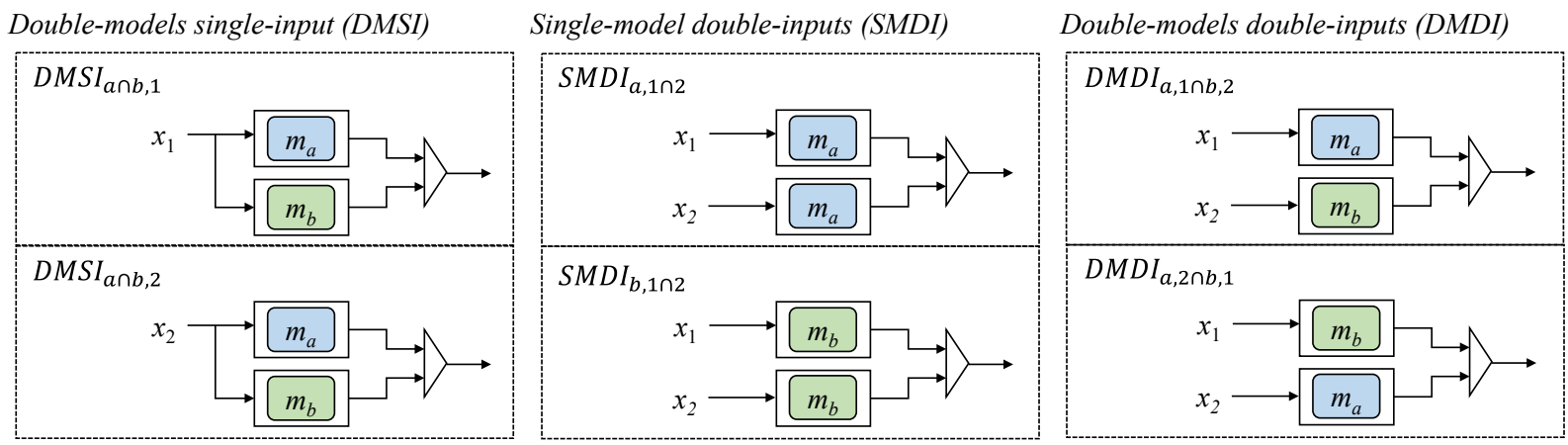

Figure 2. Architecture options for dependent double-modules double-inputs MLS

To model the reliability of MLS, we introduce the following notations. Let $x_{i}, i=\{1,2, \ldots\}$ be an input data set from a sensor $i$ and let $m_{j}, j=\{a, b, \ldots\}$ be a ML model. An ML module is a unit of the MLS, which installs one ML model $m_{j}$ and selects one input data set $x_{i}$. The ML module outputs errors when the installed ML model does not classify the input data correctly. Let $S$ be the total set of possible input data and let $E_{j} \in S$ be the subset of $S$ that makes ML model $m_{j}$ outputs errors. The probability that the module outputs error can be represented by $P\left[x_{i} \in E_{j}\right]$. Therefore, the reliability of MLS using this ML module solely is given by $1-P\left[x_{i} \in E_{j}\right]$. Throughout this paper, we assume $P\left[x_{i} \in E_{j}\right] \in(0,1)$ unless otherwise stated. The possibility that the input $x_{i}$ occurs in a real environment can also depend on the sensor's capability. To consider the randomness of sensor input, denote $X_{i}$ as the random variable representing the input data $x_{i}$ and define $\mu_{X_{i}}\left(x_{i}\right)$ as the corresponding distribution function. When we define error function $f_{j}$ as

$$
f_{j}\left(x_{i}\right)= \begin{cases}1, & x_{i} \in E_{j} \\ 0, & \text { otherwise, }\end{cases}
$$

the error probability can be expressed as

$$
P\left[x_{i} \in E_{j}\right]=\int f_{j}\left(x_{i}\right) d \mu_{X_{i}}\left(x_{i}\right) .
$$

Note that $P\left[x_{i} \in E_{j}\right]$ is the expectation of the value of $f_{j}$ for given input data distribution, and the value should be in $(0,1)$. $f_{j}$ is also called score function and used for representing the reliability of multi-version software systems [37][38]. We will use these notations in the following discussion of reliability models.

\section{Double-modules double-inputs MLS}

With the reliability function defined above, next, we formulate the reliability of MLS with redundant configuration. Given two different sensor inputs $x_{1}$ and $x_{2}$ and two different ML models $m_{a}$ and $m_{b}$, there could be six different architecture choices which are categorized into three types, as shown in Figure 2. Note that no matter which architecture is adopted, the final output of the MLS is determined by voting of two modules' outputs. Voting strategies are commonly adopted in multi-version machine learning systems [5][26][36]. In our study, we assume that the MLS outputs the prediction results only when two modules output an identical result. Therefore, we consider the reliability of system output by the probability that both two modules' outputs are correct. If any one of the modules outputs an error, the voter cannot judge which output is correct and hence discard all the outputs. The system may issue an alert to higher-level modules or users when encountering such conditions several times. We do not consider this case as a system failure in this paper. The reliabilities of the six architectures are characterized by the input data distributions and error functions as formulated below.

\section{Double-models single-input (DMSI) architecture}

In this architecture, two ML modules employ different ML models while they choose the same input data. Depending on which input data $\left(x_{1}\right.$ or $\left.x_{2}\right)$ is chosen, there are two architecture options. The reliabilities of these architectures can be represented by

$$
\begin{aligned}
& R_{D M S I_{a \cap b, 1}}=1-P\left[x_{1} \in E_{a} \cap E_{b}\right], \\
& R_{D M S I_{a \cap b, 2}}=1-P\left[x_{2} \in E_{a} \cap E_{b}\right] .
\end{aligned}
$$

Given the input data distributions $\mu_{X_{1}}\left(x_{1}\right)$ and $\mu_{X_{2}}\left(x_{2}\right)$, the reliabilities can also be expressed as

$$
\begin{aligned}
& R_{D M S I_{a \cap b, 1}}=1-\int f_{a}\left(x_{1}\right) f_{b}\left(x_{1}\right) d \mu_{X_{1}}\left(x_{1}\right), \\
& R_{D M S I_{a \cap b, 2}}=1-\int f_{a}\left(x_{2}\right) f_{b}\left(x_{2}\right) d \mu_{X_{2}}\left(x_{2}\right) .
\end{aligned}
$$

\section{Single-model double-inputs (SMDI) architecture}

In this architecture, both ML modules employ the same ML model but choose different sensor input data resulting in two potentially different outputs. Depending on which ML model $\left(m_{a}\right.$ or $\left.m_{b}\right)$ is employed, there are two architecture options. The reliabilities of these architectures can be represented by

$$
\begin{aligned}
& R_{S M D I_{a, 1 \cap 2}}=1-P\left[x_{1} \in E_{a}, x_{2} \in E_{a}\right], \\
& R_{S M D I_{b, 1 \cap 2}}=1-P\left[x_{1} \in E_{b}, x_{2} \in E_{b}\right] .
\end{aligned}
$$

Let $\mu_{X_{1}, X_{2}}\left(x_{1}, x_{2}\right)$ be the joint distribution function of two input data. The reliabilities can also be expressed as

$$
\begin{aligned}
& R_{S M D I_{a, 1 \cap 2}}=1-\int f_{a}\left(x_{1}\right) f_{a}\left(x_{2}\right) d \mu_{X_{1}, X_{2}}\left(x_{1}, x_{2}\right), \\
& R_{S M D I_{b, 1 \cap 2}}=1-\int f_{b}\left(x_{1}\right) f_{b}\left(x_{2}\right) d \mu_{X_{1}, X_{2}}\left(x_{1}, x_{2}\right) .
\end{aligned}
$$

\section{Double-models double-inputs (DMDI) architecture}

This architecture uses two different inputs and two different ML models. Two ML modules deploy different ML models and choose different sensor inputs. Depending on the 
combination of input data and ML model, there are two architecture options in this case as well. The reliabilities of these architectures can be represented by

$$
\begin{aligned}
& R_{D M D I_{a, 1 n b, 2}}=1-P\left[x_{1} \in E_{a}, x_{2} \in E_{b}\right], \\
& R_{D M D I_{a, 2 n b, 1}}=1-P\left[x_{2} \in E_{a}, x_{1} \in E_{b}\right] .
\end{aligned}
$$

With the joint distribution function of two input data, the reliabilities can also be expressed as

$$
\begin{aligned}
& R_{D M D I_{a, 1 \cap b, 2}}=1-\int f_{a}\left(x_{1}\right) f_{b}\left(x_{2}\right) d \mu_{X_{1}, X_{2}}\left(x_{1}, x_{2}\right), \\
& R_{D M D I_{a, 2 \cap b, 1}}=1-\int f_{b}\left(x_{1}\right) f_{a}\left(x_{2}\right) d \mu_{X_{1}, X_{2}}\left(x_{1}, x_{2}\right) .
\end{aligned}
$$

The architecture reliability comparison is easy if we can assume two modules' outputs are independent of each other. When we know the reliability of individual modules' outputs, the reliability of redundant configuration can be simply computed by combinatorial reliability models such as reliability block diagram [11] or fault tree [12][13]. However, the independence assumptions on the input data distributions and the errors of different ML models are unlikely to hold in practice. Moreover, we hardly obtain complete knowledge about the degrees of dependence between two ML models and two input data sets [6]. The architecture reliability comparison under such conditions is not a trivial issue.

\section{Problem}

With the above notations of MLS architectures' reliability, the general problem we try to address in this paper can be described as follows.

MLS redundant configuration problem: Given two different sensor inputs $x_{1}, x_{2}$ and two different $M L$ models $m_{a}, m_{b}$ for the same classification task, determine the best or preferable architecture options in terms of system reliability without knowing the complete information about the input data distributions and error input sets of the ML models.

Answers to this problem must give guides to choose the relevant configuration of redundant MLS architecture. Since we do not have complete knowledge about the input data distributions or the errors of ML models, the best option may not be determined due to the lack of information. However, any additional information may help to screen inappropriate options in terms of system reliability. Finding preferable architecture options from any available information is practically meaningful in the design of reliable MLS.

\section{DIVERSITY MEASURES AND RELIABILITY MODEL}

Instead of directly approaching the input data distributions and errors input sets of ML models, we attempt to characterize the architecture reliability through the diversities among the modules. In this section, we define two diversity measures and formulate the architecture reliabilities in a compact matrix representation form.

\section{A. Diversity measures}

Input data from different sensors must be very similar since both the sensors observe the same target. On the other hand, error tendencies of different ML models must be similar since both the models are trained for the same task and possibly trained from the same data sets. The degree of these similarities must influence the system reliability and may bring useful information to determine the preferable architecture option. To incorporate the factor of dependence quantitatively in the reliability model, we introduce two measures of diversity.

Intersection of errors (model similarity): Let $E_{a}$ and $E_{b}$ be the subsets of input space $S$ that make machine learning models $m_{a}$ and $m_{b}$ output errors, respectively. For an input data $x_{i}$ sampled from distribution $\mu_{X_{i}}\left(x_{i}\right)$, the intersection of errors $\alpha_{b \mid a, i}$ is defined by the conditional probability

$$
\alpha_{b \mid a, i}=P\left[x_{i} \in E_{b} \mid x_{i} \in E_{a}\right]=\frac{P\left[x_{i} \in E_{a} \cap E_{b}\right]}{P\left[x_{i} \in E_{a}\right]},
$$

where $P\left[x_{i} \in E_{a}\right]>0$.

The intersection of errors represents the degree of overlap between the sets $E_{a}$ and $E_{b}$. The larger the elements of $E_{b}$ overlaps the elements of $E_{a}$, the value of $\alpha_{b \mid a, i}$ becomes larger. Since $E_{a}$ and $E_{b}$ are attributed by the capabilities of different ML models, their intersection indicates how two models are like each other in terms of error input space. The smaller intersection decreases the probability of common errors of $m_{a}$ and $m_{b}$, which corresponds to the smaller model similarity.

Conjunction of errors (input similarity): Let $x_{1}$ and $x_{2}$ be the input data that follow distributions $\mu_{X_{1}}\left(x_{1}\right)$ and $\mu_{X_{2}}\left(x_{2}\right)$, respectively. For a machine learning model $m_{j}$ whose error space is given by $E_{j}$, the conjunction of errors $\beta_{j, 2 \mid 1}$ is defined by the conditional probability

$$
\beta_{j, 2 \mid 1}=\operatorname{Pr}\left[x_{2} \in E_{j} \mid x_{1} \in E_{j}\right]=\frac{P\left[x_{1} \in E_{j}, x_{2} \in E_{j}\right]}{P\left[x_{1} \in E_{j}\right]} .
$$

where $P\left[x_{1} \in E_{j}\right]>0$.

The conjunction of errors represents the possibility of both inputs $x_{1}$ and $x_{2}$ fall into error outputs by the process of the same ML model $m_{j}$. The larger similarity there is between distributions $\mu_{X_{1}}\left(x_{1}\right)$ and $\mu_{X_{2}}\left(x_{2}\right)$ in error space $E_{j}$, the value of $\beta_{j, 2 \mid 1}$ becomes the larger. The difference of distributions can be regarded as the diversity of the sensors' capabilities. The smaller conjunction decreases the probability of double errors as a result of the smaller input similarity.

With the defined diversity measures, the reliabilities of DMSI and SMDI architectures can be expressed as

$$
\begin{aligned}
R_{D M S I_{a \cap b, 1}} & =1-\alpha_{b \mid a, 1} \cdot P\left[x_{1} \in E_{a}\right] \\
& =1-\alpha_{a \mid b, 1} \cdot P\left[x_{1} \in E_{b}\right], \\
R_{S M D I_{a, 1 \cap 2}} & =1-\beta_{a, 2 \mid 1} \cdot P\left[x_{1} \in E_{a}\right] \\
& =1-\beta_{a, 1 \mid 2} \cdot P\left[x_{2} \in E_{a}\right] .
\end{aligned}
$$

Although the reliabilities of DMDI architectures are not simply expressed by these diversity measures, obviously, they are also affected by these diversities. We will investigate this relation in the following section.

\section{B. Reliability model for DMDI}

Since DMDI architecture uses two sensor input data and two 
distinct ML models, both model diversity and input diversity can influence the architecture reliability. Let us first consider the reliability of $D M D I_{a, 1 \cap b, 2}$. The probability that two modules in $D M D I_{a, 1 \cap b, 2}$ output errors simultaneously is given by $P\left[x_{1} \in E_{a}, x_{2} \in E_{b}\right]$. Assume that the input $x_{1}$ causes an error of $m_{a}$, the error probability of MLS can be given by conditioning whether error conjunction occurs or not;

$$
\begin{aligned}
& P\left[x_{1} \in E_{a}, x_{2} \in E_{b}\right]= \\
& P\left[x_{2} \in E_{b} \mid x_{2} \in E_{a}, x_{1} \in E_{a}\right] \cdot P\left[x_{2} \in E_{a} \mid x_{1} \in E_{a}\right] \\
& +P\left[x_{2} \in E_{b} \mid x_{2} \in \overline{E_{a}}, x_{1} \in E_{a}\right] \cdot P\left[x_{2} \in \overline{E_{a}} \mid x_{1} \in E_{a}\right] \\
& \cdot P\left[x_{1} \in E_{a}\right],
\end{aligned}
$$

where $\bar{E}=S \backslash E$ represents the complementary set of $E$ and $P\left[x_{2} \in E_{a} \mid x_{1} \in E_{a}\right] \in(0,1)$. The first term of the above expression corresponds to the probability that $x_{2}$ occurs in $E_{a} \cap E_{b}$, while the second term corresponds to the probability that $x_{2}$ occurs in $\overline{E_{a}} \cap E_{b}$. Because $\mathrm{P}\left[x_{2} \in \overline{E_{a}} \mid x_{1} \in E_{a}\right]$ complements $\mathrm{P}\left[x_{2} \in E_{a} \mid x_{1} \in E_{a}\right]$, using the input diversity $\beta_{a, 2 \mid 1}$ we can rewrite the expression

$P\left[x_{1} \in E_{a}, x_{2} \in E_{b}\right]=$

$$
\begin{aligned}
& P\left[x_{2} \in E_{b} \mid x_{2} \in E_{a}, x_{1} \in E_{a}\right] \cdot \beta_{a, 2 \mid 1} \cdot P\left[x_{1} \in E_{a}\right] \\
+ & P\left[x_{2} \in E_{b} \mid x_{2} \in \overline{E_{a}}, x_{1} \in E_{a}\right] \cdot\left(1-\beta_{a, 2 \mid 1}\right) \cdot P\left[x_{1} \in E_{a}\right] .
\end{aligned}
$$

When we define

$$
\begin{aligned}
& \alpha_{b, 2 \mid a, 1 \cap 2}=P\left[x_{2} \in E_{b} \mid x_{2} \in E_{a}, x_{1} \in E_{a}\right], \\
& \alpha_{b, 2 \mid a, 1 \cap \overline{2}}=P\left[x_{2} \in E_{b} \mid x_{2} \in \overline{E_{a}}, x_{1} \in E_{a}\right],
\end{aligned}
$$

the reliability of this architecture can be expressed as

$$
\begin{gathered}
R_{D M D I_{a, 1 \cap b, 2}}=1-\left[\alpha_{b, 2 \mid a, 1 \cap 2} \cdot \beta_{a, 2 \mid 1}+\alpha_{b, 2 \mid a, 1 \cap \overline{2}}\right. \\
\left.\cdot\left(1-\beta_{a, 2 \mid 1}\right)\right] \cdot P\left[x_{1} \in E_{a}\right] .
\end{gathered}
$$

When the intersection of errors $\alpha_{b \mid a, 2}=P\left[x_{2} \in E_{b} \mid x_{2} \in E_{a}\right]$ increases, the value of $\alpha_{b, 2 \mid a, 1 \cap 2}$ also increases while the value of $\alpha_{b, 2 \mid a, 1 \cap \overline{2}}$ decreases. Expression (1) characterizes the reliability of $D M D I_{a, 1 \cap b, 2}$ by the combination of model similarity and input similarity. However, expression (1) does not teach anything about the preferable similarity values to improve the reliability value. Without any additional information about the input data distributions, little can be learned only from this formula. Meanwhile, we can argue the relationship between different architecture options through the set of diversity-associated parameters, which is discussed in the subsequent subsection.

\section{Matrix representation}

In the derivation of the reliability of $D M D I_{a, 1 \cap b, 2}$ above, we condition the error probability by the occurrence of error conjunction to $x_{1} \in E_{a}$. Applying the same conditioning to the expression for the reliability of $D M S I_{a \cap b, 1}$, we have

$$
\begin{aligned}
& P\left[x_{1} \in E_{a}, x_{1} \in E_{b}\right]= \\
& P\left[x_{1} \in E_{b} \mid x_{2} \in E_{a}, x_{1} \in E_{a}\right] \cdot \mathrm{P}\left[x_{2} \in E_{a} \mid x_{1} \in E_{a}\right] \\
& +P\left[x_{1} \in E_{b} \mid x_{2} \in \overline{E_{a}}, x_{1} \in E_{a}\right] \cdot \mathrm{P}\left[x_{2} \in \overline{E_{a}} \mid x_{1} \in E_{a}\right] \\
& \cdot P\left[x_{1} \in E_{a}\right],
\end{aligned}
$$

where $P\left[x_{2} \in E_{a} \mid x_{1} \in E_{a}\right] \in(0,1)$. When we define

$$
\begin{aligned}
& \alpha_{b, 1 \mid a, 1 \cap 2}=P\left[x_{1} \in E_{b} \mid x_{2} \in E_{a}, x_{1} \in E_{a}\right], \\
& \alpha_{b, 1 \mid a, 1 \cap \overline{2}}=P\left[x_{1} \in E_{b} \mid x_{2} \in \overline{E_{a}}, x_{1} \in E_{a}\right],
\end{aligned}
$$

the reliability of $D M S I_{a \cap b, 1}$ can be expressed as

$$
\begin{aligned}
R_{D M S I} I_{a \cap b, 1}=1- & {\left[\alpha_{b, 1 \mid a, 1 \cap 2} \cdot \beta_{a, 2 \mid 1}+\alpha_{b, 1 \mid a, 1 \cap \overline{2}}\right.} \\
& \left.\cdot\left(1-\beta_{a, 2 \mid 1}\right)\right] \cdot P\left[x_{1} \in E_{a}\right] .
\end{aligned}
$$

The value of $R_{D M S I_{a \cap b, 1}}$ is monotonically increasing as the intersection of errors $\alpha_{b \mid a, 2}=P\left[x_{2} \in E_{b} \mid x_{2} \in E_{a}\right]$ decreases.

In a similar manner, the reliabilities of $D M D I_{a, 2 \cap b, 1}$ and $D M S I_{a \cap b, 2}$ are derived by conditioning whether error conjunction occurs at $x_{2} \in E_{a}$,

$$
\begin{aligned}
R_{D M D I_{a, 2 \cap b, 1}}=1- & {\left[\alpha_{b, 1 \mid a, 1 \cap 2} \cdot \beta_{a, 1 \mid 2}+\alpha_{b, 1 \mid a, \overline{1} \cap 2}\right.} \\
\cdot & \left.\left(1-\beta_{a, 1 \mid 2}\right)\right] \cdot P\left[x_{2} \in E_{a}\right], \\
R_{D_{M S I} a n b, 2}=1- & {\left[\alpha_{b, 2 \mid a, 1 \cap 2} \cdot \beta_{a, 1 \mid 2}+\alpha_{b, 2 \mid a, \overline{1} \cap 2}\right.} \\
\cdot & \left.\cdot\left(1-\beta_{a, 1 \mid 2}\right)\right] \cdot P\left[x_{2} \in E_{a}\right],
\end{aligned}
$$

where

$$
\begin{aligned}
& \alpha_{b, 1 \mid a, \overline{1} \cap 2}=P\left[x_{1} \in E_{b} \mid x_{1} \in \overline{E_{a}}, x_{2} \in E_{a}\right], \\
& \alpha_{b, 2 \mid a, \overline{1} \cap 2}=P\left[x_{2} \in E_{b} \mid x_{1} \in \overline{E_{a}}, x_{2} \in E_{a}\right] .
\end{aligned}
$$

The expressions (1)-(4) can be merged into the following matrix representation

where

$$
\boldsymbol{R}_{b \mid a}=\boldsymbol{J}_{2}-\boldsymbol{A}_{b \mid a} \cdot \boldsymbol{B}_{a}^{\top} \cdot \boldsymbol{P}_{a}
$$

$$
\begin{aligned}
& \boldsymbol{R}_{b \mid a}=\left[\begin{array}{cc}
R_{D M S I_{a n b, 1}} & R_{D M D I_{a, 2 n b, 1}} \\
R_{D I_{a, 1 \cap b, 2}} & R_{D M S I_{a \cap b, 2}}
\end{array}\right], \quad J_{2}=\left[\begin{array}{ll}
1 & 1 \\
1 & 1
\end{array}\right], \\
& \boldsymbol{A}_{b \mid a}=\left[\begin{array}{lll}
\alpha_{b, 1 \mid a, 1 \cap 2} & \alpha_{b, 1 \mid a, 1 \cap \overline{2}} & \alpha_{b, 1 \mid a, \overline{1} \cap 2} \\
\alpha_{b, 2 \mid a, 1 \cap 2} & \alpha_{b, 2 \mid a, 1 \cap \overline{2}} & \alpha_{b, 2 \mid a, \overline{1} \cap 2}
\end{array}\right], \\
& \boldsymbol{B}_{a}=\left[\begin{array}{ccc}
\beta_{a, 2 \mid 1} & 1-\beta_{a, 2 \mid 1} & 0 \\
\beta_{a, 1 \mid 2} & 0 & 1-\beta_{a, 1 \mid 2}
\end{array}\right], \\
& \boldsymbol{P}_{a}=\left[\begin{array}{cc}
P\left[x_{1} \in E_{a}\right] & 0 \\
0 & P\left[x_{2} \in E_{a}\right]
\end{array}\right] .
\end{aligned}
$$

The matrix $\boldsymbol{R}_{b \mid a}$ represents the reliabilities of four different architectures. $\boldsymbol{J}_{2}$ is a two-dimensional all-ones matrix. The elements of $\boldsymbol{A}_{b \mid a}$ are associated with the intersection of errors in $E_{b}$ over $E_{a}$, while the elements of $\boldsymbol{B}_{a}$ represents the conjunction or non-conjunction of errors in $E_{a}$, and $\boldsymbol{P}_{a}$ represents the error probabilities on $E_{a}$ by inputs $x_{1}$ and $x_{2}$.

The matrix term $\boldsymbol{B}_{a}^{\top} \cdot \boldsymbol{P}_{a}$ in (5) can be represented by the functions of $R_{S M D I_{a, 1 \cap 2}}$ as follows;

$$
\begin{aligned}
& \boldsymbol{B}_{a}^{\top} \cdot \boldsymbol{P}_{a} \\
& =\left[\begin{array}{cc}
\beta_{a, 2 \mid 1} \cdot P\left[x_{1} \in E_{a}\right] & \beta_{a, 1 \mid 2} \cdot P\left[x_{2} \in E_{a}\right] \\
\left(1-\beta_{a, 2 \mid 1}\right) \cdot P\left[x_{1} \in E_{a}\right] & 0 \\
0 & \left(1-\beta_{a, 1 \mid 2}\right) \cdot P\left[x_{2} \in E_{a}\right]
\end{array}\right] \\
& =\left[\begin{array}{cc}
1-R_{S M D I_{a, 1 \cap 2}} & 1-R_{S M D I_{a, 1 \cap 2}} \\
P\left[x_{1} \in E_{a}\right]+R_{S M D I_{a, 1 \cap 2}}-1 & 0 \\
0 & P\left[x_{2} \in E_{a}\right]+R_{S M D I_{a, 1 \cap 2}}-1
\end{array}\right] .
\end{aligned}
$$

Therefore, the expression (5) characterizes the relationships among the reliabilities of five different architectures out of six options (i.e, only $R_{S M D I_{b, 1 \cap 2}}$ is not associated).

A similar derivation can be carried out by conditioning the error conjunction or non-conjunction in $E_{b}$. As a result, the dual of $\boldsymbol{R}_{b \mid a}$ can be obtained by exchanging $E_{a}$ and $E_{b}$ in (5),

$$
\boldsymbol{R}_{a \mid b}=\boldsymbol{J}_{2}-\boldsymbol{A}_{a \mid b} \cdot \boldsymbol{B}_{b}^{\top} \cdot \boldsymbol{P}_{b},
$$

where 


$$
\begin{aligned}
\boldsymbol{R}_{a \mid b} & =\left[\begin{array}{cc}
R_{D M S I_{a n b, 1}} & R_{D M D I_{a, 1 \cap b, 2}} \\
R_{D M D I_{a, 2 \cap b, 1}} & R_{D M S I_{a n b, 2}}
\end{array}\right], \\
\boldsymbol{A}_{a \mid b} & =\left[\begin{array}{lll}
\alpha_{a, 1 \mid b, 1 \cap 2} & \alpha_{a, 1 \mid b, 1 \cap \overline{2}} & \alpha_{a, 1 \mid b, \overline{1} \cap 2} \\
\alpha_{a, 2 \mid b, 1 \cap 2} & \alpha_{a, 2 \mid b, 1 \cap \overline{2}} & \alpha_{a, 2 \mid b, \overline{1} \cap 2}
\end{array}\right], \\
\boldsymbol{B}_{b}= & {\left[\begin{array}{ccc}
\beta_{b, 2 \mid 1} & 1-\beta_{b, 2 \mid 1} & 0 \\
\beta_{b, 1 \mid 2} & 0 & 1-\beta_{b, 1 \mid 2}
\end{array}\right], } \\
\boldsymbol{P}_{b}= & {\left[\begin{array}{cc}
P\left[x_{1} \in E_{b}\right. & 0 \\
0 & P\left[x_{2} \in E_{b}\right]
\end{array}\right] . }
\end{aligned}
$$

Since the expression (6) includes the term $\boldsymbol{B}_{b}^{\top} \cdot \boldsymbol{P}_{b}$

$$
\begin{aligned}
& =\left[\begin{array}{cc}
\beta_{b, 2 \mid 1} \cdot P\left[x_{1} \in E_{b}\right] & \beta_{b, 1 \mid 2} \cdot P\left[x_{2} \in E_{b}\right] \\
\left(1-\beta_{b, 2 \mid 1}\right) \cdot P\left[x_{1} \in E_{b}\right] & 0 \\
0 & \left(1-\beta_{b, 1 \mid 2}\right) \cdot P\left[x_{2} \in E_{b}\right]
\end{array}\right] \\
& =\left[\begin{array}{cc}
1-R_{S M D I_{b, 1 \cap 2}} & 1-R_{S M D I_{b, 1 \cap 2}} \\
P\left[x_{1} \in E_{b}\right]+R_{S M D I_{b, 1 \cap 2}}-1 & 0 \\
0 & P\left[x_{2} \in E_{b}\right]+R_{S M D I_{b, 1 \cap 2}}-1
\end{array}\right] .
\end{aligned}
$$

the relationships among the reliabilities of five different architectures out of six options are represented by (6) (i.e., only $R_{S M D I_{a, 1 \cap 2}}$ is not associated). By definition, $\boldsymbol{R}_{b \mid a}$ and $\boldsymbol{R}_{a \mid b}$ satisfy the following relation.

$$
\boldsymbol{R}_{a \mid b}=\boldsymbol{R}_{b \mid a}^{\top}
$$

Consequently, the relationships between the reliabilities of six different architectures can be characterized by diversityproperty matrixes $\boldsymbol{A}_{b \mid a}, \boldsymbol{A}_{a \mid b}, \boldsymbol{B}_{a}$ and $\boldsymbol{B}_{b}$ under the relation (7).

In the above derivation, for a given event $x_{1} \in E_{a}$, first, we assume error conjunctions in $E_{a}$ and then consider the intersection to $E_{b}$. One can also derive a similar relationship in inverse order; i.e., first assume the error occurs in the intersection of $E_{a}$ and $E_{b}$ and then consider the conjunction of errors by another input. The reliability matrices can be represented as $\boldsymbol{R}_{2 \mid 1}$ and $\boldsymbol{R}_{1 \mid 2}$ which satisfy the relation $\boldsymbol{R}_{1 \mid 2}=$ $\boldsymbol{R}_{2 \mid 1}^{\top}$. Since the derivation is mostly the same as described above, we omit to show this case for brevity.

Both the equalities $\boldsymbol{R}_{a \mid b}=\boldsymbol{R}_{b \mid a}^{\top}$ and $\boldsymbol{R}_{1 \mid 2}=\boldsymbol{R}_{2 \mid 1}^{\top}$ can fully represent the relations among six architectures' reliabilities and diversity-associated parameters.

\section{ARCHITECTURE PROPERTY ANALYSIS}

Using the reliability model (5)(6)(7), we discuss the properties of these architectures that may help the decision of relevant architecture choice in terms of MLS reliability. The exact values of diversity parameters such as $\boldsymbol{A}_{b \mid a}$ and $\boldsymbol{B}_{a}$ are not obtainable in practice. However, we can argue a preferable architecture through the analysis of the relation of these diversity values. In the following discussion, we first show the general property derived from the model (5)(6)(7), and then present some special properties that can be shown under a specific type of joint distribution for the input data set.

\section{A. General properties}

An interesting question to the presented architecture model is which architecture achieves better reliability than another architecture under specific conditions on the diversity metrics. In other words, it is interesting to know if the value of diversity metric can determine the preference of the architecture in terms of reliability. To investigate this, first consider the reliabilities difference between the elements of $\boldsymbol{R}_{b \mid a}$ and $R_{S M D I_{a, 1 \cap 2}}$. Define the reliability difference matrix by

$$
\boldsymbol{H}_{b \mid a}=\boldsymbol{R}_{b \mid a}-R_{S M D I_{a, 1 \cap 2}} \cdot \boldsymbol{J}_{2} .
$$

By definition, $R_{S M D I_{a, 1 \cap 2}}$ can be expressed either by $\beta_{a, 2 \mid 1}$. $P\left[x_{1} \in E_{a}\right]$ or by $\beta_{a, 1 \mid 2} \cdot P\left[x_{2} \in E_{a}\right]$. We can write

$$
R_{S M D I_{a, 1 \cap 2}} \cdot \boldsymbol{J}_{2}=\boldsymbol{J}_{2}-\boldsymbol{B}_{a}^{+\top} \cdot \boldsymbol{P}_{a},
$$

where

$$
\boldsymbol{B}_{a}^{+}=\left[\begin{array}{ll}
\beta_{a, 2 \mid 1} & \beta_{a, 1 \mid 2}
\end{array}\right]^{\top} \cdot\left[\begin{array}{ll}
1 & 1
\end{array}\right]=\left[\begin{array}{ll}
\beta_{a, 2 \mid 1} & \beta_{a, 2 \mid 1} \\
\beta_{a, 1 \mid 2} & \beta_{a, 1 \mid 2}
\end{array}\right] .
$$

Applying (5) and (9) to (8),

$$
\begin{aligned}
& \boldsymbol{H}_{b \mid a}=\boldsymbol{J}_{2}-\boldsymbol{A}_{b \mid a} \cdot \boldsymbol{B}_{a}^{\top} \cdot \boldsymbol{P}_{a}-\left(\boldsymbol{J}_{2}-\boldsymbol{B}_{a}^{+\top} \cdot \boldsymbol{P}_{a}\right) \\
& =\left(\boldsymbol{B}_{a}^{+\top}-\boldsymbol{A}_{b \mid a} \cdot \boldsymbol{B}_{a}^{\top}\right) \cdot \boldsymbol{P}_{a} .
\end{aligned}
$$

The expression (10) shows that the conditions where DMDI and DMSI architectures achieve higher reliability than $R_{S M D I_{a, 1 \cap 2}}$ can be provided by the sign of $\boldsymbol{B}_{a}^{+\top}-\boldsymbol{A}_{b \mid a} \cdot \boldsymbol{B}_{a}^{\top}$. Thus, we have the general property which characterizes the reliability difference of the architectures as described next.

Lemma 1. Given a parameter matrix $\boldsymbol{A}_{b \mid a}$, the reliabilities of $D M S I_{a \cap b, 1}$ and $D M D I_{a, 1 \cap b, 2}$ monotonically increase against the reliability of $S M D I_{a, 1 \cap 2}$ as $\beta_{a, 2 \mid 1}$ increases, while the reliabilities of $D M D I_{a, 2 \cap b, 1}$ and $D M S I_{a \cap b, 2}$ monotonically increase against the reliability of $S M D I_{a, 1 \cap 2}$ as $\beta_{a, 1 \mid 2}$ increases.

The proof of the lemma is presented in Appendix. The lemma implies that the architectures employing double models (i.e., DMDI and DMSI) tend to be preferable compared with the single model architecture (i.e., SMDI) when input $x_{1}$ has higher conjunction with $x_{2}$ in $E_{a}$. Note that the condition where the double-models architecture become preferable to single model architecture is given by $\boldsymbol{H}_{b \mid a}>0$. In order to investigate the condition, we need to understand the lower and upper bound of $\boldsymbol{H}_{b \mid a}$ with respect to $\beta_{a, 2 \mid 1}$ and $\beta_{a, 1 \mid 2}$, which are given in the following lemma.

Lemma 2. Given a parameter matrix $\boldsymbol{A}_{b \mid a}$, the lower and upper bounds of $\boldsymbol{H}_{b \mid a}$ as the functions of $\beta_{a, 2 \mid 1}, \beta_{a, 1 \mid 2} \in(0,1)$ are given by

$$
\begin{aligned}
\inf \boldsymbol{H}_{b \mid a}= & {\left[\begin{array}{ll}
-\alpha_{b, 1 \mid a, 1 \cap \overline{2}} & -\alpha_{b, 1 \mid a, \overline{1} \cap 2} \\
-\alpha_{b, 2 \mid a, 1 \cap \overline{2}} & \left.-\alpha_{b, 2 \mid a, \overline{1} \cap 2}\right] \cdot \boldsymbol{P}_{a},
\end{array}\right.} \\
\sup \boldsymbol{H}_{b \mid a} & = \begin{cases}\boldsymbol{H}_{b \mid a}^{\text {sup }} \cdot \boldsymbol{P}_{a}, & P\left[x_{2} \in E_{a}\right]<P\left[x_{1} \in E_{a}\right] \\
\boldsymbol{H}_{b \mid a}^{\text {sup2 }} \cdot \boldsymbol{P}_{a}, & P\left[x_{2} \in E_{a}\right] \geq P\left[x_{1} \in E_{a}\right],\end{cases} \\
\boldsymbol{H}_{b \mid a}^{\text {sup1 }} & =\left[\begin{array}{ll}
\breve{\alpha}_{1} \cdot \frac{P\left[x_{2} \in E_{a}\right]}{P\left[x_{1} \in E_{a}\right]}-\alpha_{b, 1 \mid a, 1 \cap \overline{2}} \cdot \frac{P\left[x_{2} \in \overline{E_{a}}\right]}{P\left[x_{1} \in E_{a}\right]} & \breve{\alpha}_{1} \\
\breve{\alpha}_{2} \cdot \frac{P\left[x_{2} \in E_{a}\right]}{P\left[x_{1} \in E_{a}\right]}-\alpha_{b, 2 \mid a, 1 \cap \overline{2}} \cdot \frac{P\left[x_{2} \in \overline{E_{a}}\right]}{P\left[x_{1} \in E_{a}\right]} & \breve{\alpha}_{2}
\end{array}\right], \\
\boldsymbol{H}_{b \mid a}^{\text {sup2 }} & =\left[\begin{array}{lll}
\breve{\alpha}_{1} & \breve{\alpha}_{1} \cdot \frac{P\left[x_{1} \in E_{a}\right]}{P\left[x_{2} \in E_{a}\right]}-\alpha_{b, 1 \mid a, \overline{1} \cap 2} \cdot \frac{P\left[x_{1} \in \bar{E}_{a}\right]}{P\left[x_{2} \in E_{a}\right]} \\
\breve{\alpha}_{2} & \breve{\alpha}_{2} \cdot \frac{P\left[x_{1} \in E_{a}\right]}{P\left[x_{2} \in E_{a}\right]}-\alpha_{b, 2 \mid a, \overline{1} \cap 2} \cdot \frac{P\left[x_{1} \in \bar{E}_{a}\right]}{P\left[x_{2} \in E_{a}\right]}
\end{array}\right] .
\end{aligned}
$$

where $\breve{\alpha}_{1}=1-\alpha_{b, 1 \mid a, 1 \cap 2}$ and $\breve{\alpha}_{2}=1-\alpha_{b, 2 \mid a, 1 \cap 2}$. 
The proof is presented in Appendix. Since all the elements of the lower bound $\inf \boldsymbol{H}_{b \mid a}$ are negative, double-model architectures achieve lower reliabilities than the single-model architecture at the lower bound. By Lemma 1 the values of $\boldsymbol{H}_{b \mid a}$ increases monotonically in terms of $\beta_{a, 2 \mid 1}$ and $\beta_{a, 1 \mid 2}$, the sign of $\sup \boldsymbol{H}_{b \mid a}$ determines changes of the preference. From Lemma 1 and Lemma 2, we have the following proposition.

Proposition 1. Given a parameter matrix $\boldsymbol{A}_{b \mid a}$, when $(i, j)$ element of $\sup \boldsymbol{H}_{b \mid a}$ is positive, there exists a unique changing point on the increasing value of $\beta_{a, 2 \mid 1}$ (when $j=1$ ) or $\beta_{a, 1 \mid 2}$ (when $j=2$ ) at which the reliability of $S M D I_{a, 1 \cap 2}$ becomes lower than the reliability of $\operatorname{DMSI}_{a \cap b, 1}(i, j=1)$, $D M D I_{a, 1 \cap b, 2} \quad(i=2, \quad j=1), \quad D M D I_{a, 2 \cap b, 1} \quad(i=1, \quad j=2)$, or $D M S I_{a \cap b, 2}(i, j=2)$.

The values of $\beta_{a, 2 \mid 1}$ and $\beta_{a, 1 \mid 2}$ that give the changing points satisfy $\boldsymbol{H}_{b \mid a}=0$. From (10), the condition can also be derived from the equation $\boldsymbol{B}_{a}^{+\top}=\boldsymbol{A}_{b \mid a} \cdot \boldsymbol{B}_{a}^{\top}$.

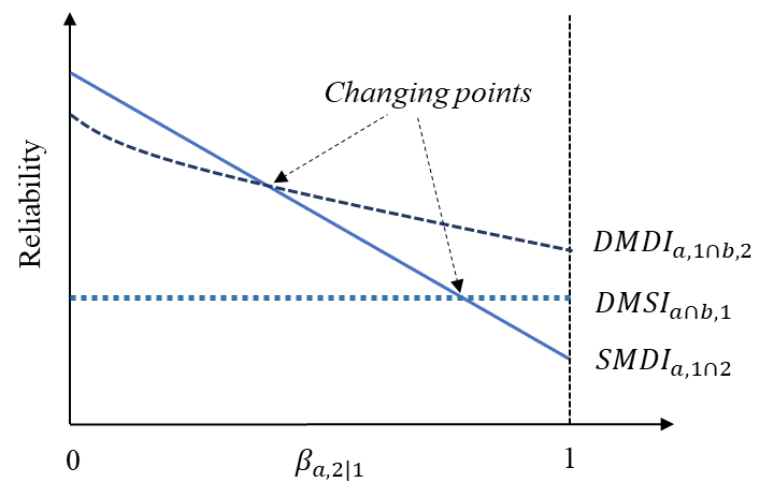

Figure 3. The example showing that the reliability of $S M D I_{a, 1 \cap 2}$ decreases as the value of $\beta_{a, 2 \mid 1}$ increase

Figure 3 shows an illustrative example of Proposition 1. The reliability of $S M D I_{a, 1 \cap 2}$ decreases by the increased $\beta_{a, 2 \mid 1}$, representing the higher conjunctions of errors. While $S M D I_{a, 1 \cap 2}$ achieves the highest reliability when $\beta_{a, 2 \mid 1}$ is low, it drops to the least option when $\beta_{a, 2 \mid 1}$ is close to one. The implication is that $S M D I_{a, 1 \cap 2}$ is a preferable architecture when we can obtain any confidence in the small value of $\beta_{a, 2 \mid 1}$ (i.e., error conjunction of inputs $x_{2}$ with inputs $x_{1}$ seldom occurs).

A similar property can be derived for the elements of $\boldsymbol{R}_{a \mid b}$ which are compared with $R_{S M D I_{b, 1 \cap 2}}$. Define the reliability difference matrix by

$$
\boldsymbol{H}_{a \mid b}=\boldsymbol{R}_{a \mid b}-R_{S M D I_{b, 1 \cap 2}} \cdot \boldsymbol{J}_{2} .
$$

The matrix can be represented by

$$
\boldsymbol{H}_{a \mid b}=\left(\boldsymbol{B}_{b}^{+\top}-\boldsymbol{A}_{a \mid b} \cdot \boldsymbol{B}_{b}^{\top}\right) \cdot \boldsymbol{P}_{b}
$$

where

$$
\boldsymbol{B}_{b}^{+}=\left[\begin{array}{ll}
\beta_{b, 2 \mid 1} & \beta_{b, 1 \mid 2}
\end{array}\right]^{\top} \cdot\left[\begin{array}{ll}
1 & 1
\end{array}\right]=\left[\begin{array}{ll}
\beta_{b, 2 \mid 1} & \beta_{b, 2 \mid 1} \\
\beta_{b, 1 \mid 2} & \beta_{b, 1 \mid 2}
\end{array}\right] .
$$

Note that (12) represents the same relation with (10), only by substituting $a$ with $b$. Consequently, the duals of Lemma 1, Lemma 2, and Proposition 1 can be shown as well.

Furthermore, the difference of elements in $\boldsymbol{R}_{2 \mid 1}$ and
$D M S I_{a \cap b, 1}$ can be characterized by the reliability difference matrix $\boldsymbol{H}_{2 \mid 1}=\boldsymbol{R}_{2 \mid 1}-D M S I_{a \cap b, 1} \cdot \boldsymbol{J}_{2}$, while the difference of elements in $\boldsymbol{R}_{1 \mid 2}$ and $D M S I_{a \cap b, 2}$ can be characterized by the reliability difference matrix $\boldsymbol{H}_{1 \mid 2}=\boldsymbol{R}_{1 \mid 2}-D M S I_{a \cap b, 2} \cdot \boldsymbol{J}_{2}$. For $\boldsymbol{H}_{2 \mid 1}$ and $\boldsymbol{H}_{1 \mid 2}$, by similar derivation steps, we can show the monotonicity, the bounds, and the changing points in terms of $\alpha_{b \mid a, 1}, \alpha_{a \mid b, 1}, \alpha_{b \mid a, 2}$ and $\alpha_{a \mid b, 2}$. We omit the details of the derivations for brevity.

\section{B. Properties under restricted distributions}

The previous section shows the general property on the difference of architectures' reliabilities in terms of diversity metrics without restricting the type of input data distributions. However, Proposition 1 is still insufficient to judge the preferable architecture for given conditions on the diversity measures. For example, the preference between $D M D I_{a, 1 \cap b, 2}$ and $D M D I_{a, 2 \cap b, 1}$ cannot be characterized through the property of either $\boldsymbol{H}_{b \mid a}$ or $\boldsymbol{H}_{a \mid b}$. To derive any useful information to select the architecture, we may need more information about the input data distributions. In the following, we consider three special cases where additional assumptions restricting the type of input data distributions help architecture selection.

\section{1) Case 1: Input superiority is known}

First, we assume that the input data $X_{2}$ is more error-prone than $X_{1}$ for any error space $E_{j}$, and clarify the conditions on diversity measures to decide the preferable architecture in this case. The assumption is likely to hold in practice when there is a non-trivial difference in sensors' capabilities (e.g., an old sensor always induces more errors than the new sensor). The assumption can formally be described as, for any subset $E^{*} \subseteq$ $E_{a} \cup E_{b}$, the joint distribution $\mu_{X_{1}, X_{2}}\left(x_{1}, x_{2}\right)$ satisfies $P\left[x_{1} \in\right.$ $\left.E^{*}\right] \leq P\left[x_{2} \in E^{*}\right]$. Under this assumption, we have relations

$$
\begin{aligned}
& P\left[x_{1} \in E_{a}\right] \leq P\left[x_{2} \in E_{a}\right], \\
& P\left[x_{1} \in E_{b}\right] \leq P\left[x_{2} \in E_{b}\right] .
\end{aligned}
$$

The above two inequalities also yield relations

$P\left[x_{1} \in E_{a} \cap E_{b}\right] \leq P\left[x_{1} \in E_{a}, x_{2} \in E_{b}\right] \leq P\left[x_{2} \in E_{a} \cap E_{b}\right]$, $P\left[x_{1} \in E_{a} \cap E_{b}\right] \leq P\left[x_{1} \in E_{b}, x_{2} \in E_{a}\right] \leq P\left[x_{2} \in E_{a} \cap E_{b}\right]$. Therefore, under this assumption, the architecture reliabilities satisfy

$$
R_{D M S I_{a \cap b, 1}} \geq\left(R_{D M D I_{a, 1 \cap b, 2}}, R_{D M D I_{a, 2 \cap b, 1}}\right) \geq R_{D M S I_{a \cap b, 2}} \text {. (13) }
$$

The inequality indicates that $D M D I_{a, 1 \cap b, 2}, D M D I_{a, 2 \cap b, 1}$ and $D M S I_{a \cap b, 2}$ are not considered as the best architecture option regardless of the values of diversity measures. Then, the question is which architecture would be the best among $D M S I_{a \cap b, 1}, S M D I_{a, 1 \cap 2}$ and $S M D I_{b, 1 \cap 2}$. The answer to this question is provided in the following proposition.

Proposition 2. Assume that the joint distribution $\mu_{X_{1}, X_{2}}\left(x_{1}, x_{2}\right)$ satisfies $P\left[x_{1} \in E^{*}\right] \leq P\left[x_{2} \in E^{*}\right]$ for any subset $E^{*} \subseteq E_{a} \cup$ $E_{b}$. The most reliable architecture is given by either 

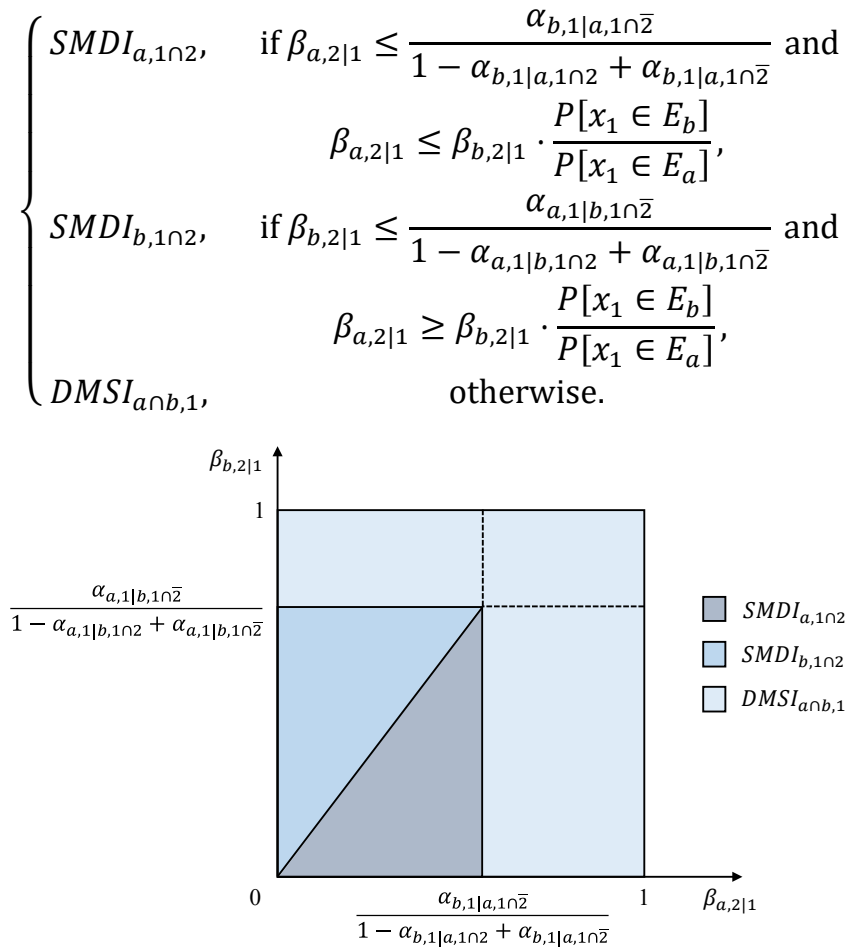

Figure 4 . Boundary conditions of the most reliable architecture

The proof of the lemma is presented in Appendix. Figure 4 visualizes the above conditions on $\left(\beta_{a, 2 \mid 1}, \beta_{b, 2 \mid 1}\right)$ coordinates. As shown in Figure 4, intuitively $D M S I_{a \cap b, 1}$ becomes the best architecture choice when we observe higher conjunction of errors (i.e., larger $\beta_{a, 2 \mid 1}$ and $\beta_{b, 2 \mid 1}$ ).

\section{2) Case 2: Model superiority is known}

Next, we assume that the errors in $E_{b}$ occurs more frequently than the errors in $E_{a}$ in any input data set and derive the conditions for deciding the preferable architecture in this case. The assumption restricts the shape of input data distributions, in particular over error spaces $E_{a}$ and $E_{b}$, while it seems relatively acceptable in practice as the error frequencies are largely dominated by the ML models' capabilities. The assumption can be described formally as; for any input data $x_{i}$ follow the input data distribution $\mu_{X_{1}}\left(x_{1}\right)$ or $\mu_{X_{2}}\left(x_{2}\right)$, it satisfies $P\left[x_{i} \in E_{a}\right] \leq P\left[x_{i} \in E_{b}\right]$. Under this assumption, we have the following relations.

$$
\begin{aligned}
& P\left[x_{1} \in E_{a}\right] \leq P\left[x_{1} \in E_{b}\right], \\
& P\left[x_{2} \in E_{a}\right] \leq P\left[x_{2} \in E_{b}\right] .
\end{aligned}
$$

The two inequalities also yield the relations

$$
\begin{array}{r}
P\left[x_{1} \in E_{a}, x_{2} \in E_{a}\right] \leq P\left[x_{1} \in E_{a}, x_{2} \in E_{b}\right] \\
\leq P\left[x_{1} \in E_{b}, x_{2} \in E_{b}\right] \\
P\left[x_{1} \in E_{a}, x_{2} \in E_{a}\right] \leq P\left[x_{1} \in E_{b}, x_{2} \in E_{a}\right] \\
\leq P\left[x_{1} \in E_{b}, x_{2} \in E_{b}\right] .
\end{array}
$$

Under this assumption, the architecture reliabilities satisfy

$$
R_{S M D I_{a, 1 \cap 2}} \geq\left(R_{D M D I_{a, 1 \cap b, 2}}, R_{D M D I_{a, 2 \cap b, 1}}\right) \geq R_{S M D I_{b, 1 \cap 2}} \text {. (14) }
$$

In this case, either $S M D I_{a, 1 \cap 2}, D M S I_{a \cap b, 1}$ or $D M S I_{a \cap b, 2}$ becomes the most reliable architecture, which can be determined by the following proposition. We omit the proof, but it can be shown using the matrix $\boldsymbol{H}_{2 \mid 1}$ and $\boldsymbol{H}_{1 \mid 2}$ in a similar derivation step as shown in the proof of Proposition 2.

Proposition 3. Assume that $P\left[x_{i} \in E_{a}\right] \leq P\left[x_{i} \in E_{b}\right]$ satisfies for any input data $x_{i}$ follow the input data distribution $\mu_{X_{1}}\left(x_{1}\right)$ or $\mu_{X_{2}}\left(x_{2}\right)$. The most reliable architecture is given by either

$$
\left\{\begin{array}{rr}
D M S I_{a \cap b, 1}, \quad \text { if } \alpha_{b \mid a, 1} \leq & \frac{\beta_{a, 2 \mid a \cap \bar{b}, 1}}{1-\beta_{a, 2 \mid a \cap b, 1}+\beta_{a, 2 \mid a \cap \bar{b}, 1}} \text { and } \\
\alpha_{b \mid a, 1} & \leq \alpha_{b \mid a, 2} \cdot \frac{P\left[x_{2} \in E_{a}\right]}{P\left[x_{1} \in E_{a}\right]}, \\
D M S I_{a \cap b, 2}, & \text { if } \alpha_{b \mid a, 2} \leq \frac{\beta_{a, 1 \mid a \cap \bar{b}, 2}}{1-\beta_{a, 1 \mid a \cap b, 2}+\beta_{a, 1 \mid a \cap \bar{b}, 2}} \text { and } \\
\alpha_{b \mid a, 1} & \geq \alpha_{b \mid a, 2} \cdot \frac{P\left[x_{2} \in E_{a}\right]}{P\left[x_{1} \in E_{a}\right]}, \\
\text { otherwise, }
\end{array}\right.
$$

where

$$
\begin{aligned}
& \beta_{a, 2 \mid a \cap b, 1}=P\left[x_{2} \in E_{a} \mid x_{1} \in E_{a}, x_{1} \in E_{b}\right], \\
& \beta_{a, 2 \mid a \cap \bar{b}, 1}=P\left[x_{2} \in E_{a} \mid x_{1} \in E_{a}, x_{1} \in \overline{E_{b}}\right], \\
& \beta_{a, 1 \mid a \cap b, 2}=P\left[x_{1} \in E_{a} \mid x_{2} \in E_{a}, x_{2} \in E_{b}\right], \\
& \beta_{a, 1 \mid a \cap \bar{b}, 2}=P\left[x_{1} \in E_{a} \mid x_{2} \in E_{a}, x_{2} \in \overline{E_{b}}\right] .
\end{aligned}
$$

Proposition 3 indicates that $S M D I_{a, 1 \cap 2}$ becomes the best architecture choice when we observe a higher intersection of errors (i.e., larger $\alpha_{b \mid a, 1}$ and $\alpha_{b \mid a, 2}$ ).

3) Case 3: Complementary models and inputs are given

In the above two possible scenarios with reasonable assumptions, we find that DMDI architectures are neither the best architecture nor the worst architecture choice. An interesting question is whether DMDI architectures can become the best option in terms of reliability under a reasonable assumption. The answer could be yes when the two input data distributions have a complementary relationship about the error-proneness of the different ML models. The assumption can be formally defined as; for any subsets $E_{a}^{*} \subseteq E_{a}$ and $E_{b}^{*} \subseteq$ $E_{b}$, the joint distribution $\mu_{X_{1}, X_{2}}\left(x_{1}, x_{2}\right)$ satisfies $P\left[x_{1} \in E_{a}^{*}\right] \leq$ $P\left[x_{2} \in E_{a}^{*}\right]$ and $P\left[x_{2} \in E_{b}^{*}\right] \leq P\left[x_{1} \in E_{b}^{*}\right]$. Under this assumption, we have

$$
\begin{aligned}
& P\left[x_{1} \in E_{a}\right] \leq P\left[x_{2} \in E_{a}\right], \\
& P\left[x_{2} \in E_{b}\right] \leq P\left[x_{1} \in E_{b}\right] .
\end{aligned}
$$

The two inequalities also yield the relations

$$
\begin{array}{r}
P\left[x_{1} \in E_{a}, x_{2} \in E_{b}\right] \leq P\left[x_{1} \in E_{a} \cap E_{b}\right] \\
\leq P\left[x_{1} \in E_{b}, x_{2} \in E_{a}\right], \\
P\left[x_{1} \in E_{a}, x_{2} \in E_{b}\right] \leq P\left[x_{2} \in E_{a} \cap E_{b}\right] \\
\leq P\left[x_{1} \in E_{b}, x_{2} \in E_{a}\right] .
\end{array}
$$

Therefore, under this assumption, the architecture reliabilities satisfy

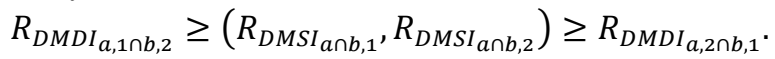

Using $\boldsymbol{H}_{b \mid a}$ and $\boldsymbol{H}_{a \mid b}$, we can show the next proposition that clarifies the conditions where $D M D I_{a, 1 \cap b, 2}$ can achieve the best reliability among other architectures.

Proposition 4. Assume that the joint distribution $\mu_{X_{1}, X_{2}}\left(x_{1}, x_{2}\right)$ satisfies $\quad P\left[x_{1} \in E_{a}^{*}\right] \leq P\left[x_{2} \in E_{a}^{*}\right] \quad$ and $P\left[x_{2} \in E_{b}^{*}\right] \leq$ $P\left[x_{1} \in E_{b}^{*}\right]$ for any subsets $E_{a}^{*} \subseteq E_{a}$ and $E_{b}^{*} \subseteq E_{b}$. The most reliable architecture is given by either one of the following. 


$$
\begin{aligned}
& \left(S M D I_{a, 1 \cap 2}, \quad \text { if } \beta_{a, 2 \mid 1} \leq \frac{\alpha_{b, 2 \mid a, 1 \cap \overline{2}}}{1-\alpha_{b, 2 \mid a, 1 \cap 2}+\alpha_{b, 2 \mid a, 1 \cap \overline{2}}}\right. \text { and } \\
& \beta_{a, 2 \mid 1} \leq \beta_{b, 1 \mid 2} \cdot \frac{P\left[x_{2} \in E_{b}\right]}{P\left[x_{1} \in E_{a}\right]}, \\
& S M D I_{b, 1 \cap 2}, \quad \text { if } \beta_{b, 1 \mid 2} \leq \frac{\alpha_{a, 1 \mid b, \overline{1} \cap 2}}{1-\alpha_{a, 1 \mid b, 1 \cap 2}+\alpha_{a, 1 \mid b, \overline{1} \cap 2}} \text { and } \\
& \beta_{a, 2 \mid 1} \geq \beta_{b, 1 \mid 2} \cdot \frac{P\left[x_{2} \in E_{b}\right]}{P\left[x_{1} \in E_{a}\right]}, \\
& D M D I_{a, 1 \cap b, 2}, \quad \text { otherwise. }
\end{aligned}
$$

Figure 5 visualizes the above conditions on $\left(\beta_{a, 2 \mid 1}, \beta_{b, 1 \mid 2}\right)$ coordinates.

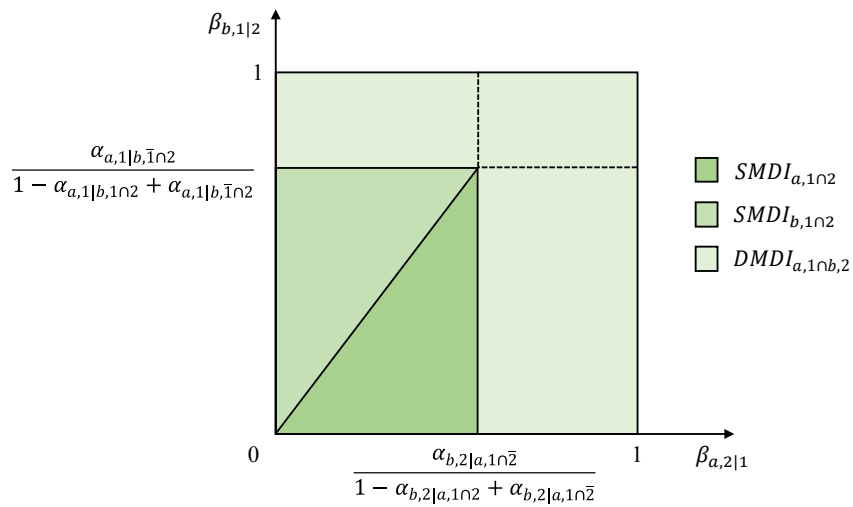

Figure 5. Conditions where DMDI architectures achieve the highest reliability

By symmetric assumption, we can also derive the conditions where $D M D I_{a, 2 \cap b, 1}$ can achieve the best reliability. Alternatively, when we assume the input data distributions $\mu_{X_{1}}\left(x_{1}\right)$ and $\mu_{X_{2}}\left(x_{2}\right)$ satisfy $P\left[x_{1} \in E_{a}\right] \leq P\left[x_{1} \in E_{b}\right]$ and $P\left[x_{2} \in E_{b}\right] \leq P\left[x_{2} \in E_{a}\right]$ for any $x_{1}$ and $x_{2}$, we can show the conditions where $D M D I_{a, 1 \cap b, 2}$ can achieve higher reliability than $D M S I_{a \cap b, 1}$ and $D M S I_{a \cap b, 2}$.

In a summary of the above discussions, we can have guides to decide the appropriate architecture in terms of reliability by restricting the shape of input data distributions. For instance, if we observe more errors from the sensor input $x_{1}$ than the input $x_{2}$, from Proposition 2, it is not encouraged to choose $D M S I_{a \cap b, 2}$ considering its reliability. In practice, the values of diversity measures are not very close to either zero or one. From (13) and (14), DMDI could be considered a neutral and conservative choice when there is not much information about the preference of input data and ML models. However, it is not a trivial decision to select $D M D I_{a, 1 \cap b, 2}$ or $D M D I_{a, 2 \cap b, 1}$ because when either one choice achieves the best reliability, the other one becomes the least reliable architecture, as seen in (15).

\section{Property under the conditional independence}

In the proposed reliability model, we used the conditional diversity parameters such as $\alpha_{b, 2 \mid a, 1 \cap 2}$ and $\alpha_{b, 2 \mid a, 1 \cap \overline{2}}$. Although they have monotonic relation to $\alpha_{b \mid a, 2}=P\left[x_{2} \in E_{b} \mid x_{2} \in E_{a}\right]$, their exact values are hard to be inferred from observations. Therefore, even if restricting the input data distributions as discussed in the previous section, it is still difficult to estimate how much the selected architecture achieves higher reliability than the others. In contrast, when we assume the independence of individual ML modules' outputs, the difference of architectures' reliabilities can be easily computed. In this section, we attempt to narrow this gap by introducing the assumption of conditional independence [35] between the different diversity measures. With the conditional independence assumption, the intersection of error spaces can be considered independently of the effects of error conjunctions. This means $\alpha_{b, 2 \mid a, 1 \cap 2}=P\left[x_{2} \in E_{b} \mid x_{2} \in E_{a}, x_{1} \in E_{a}\right]$ becomes equal to $\alpha_{b \mid a, 2}=P\left[x_{2} \in E_{b} \mid x_{2} \in E_{a}\right]$, since the occurrence of the event $x_{1} \in E_{a}$ does not affect the conditional event $P\left[x_{2} \in E_{b} \mid x_{2} \in E_{a}\right]$. Assuming that $P\left[x_{2} \in E_{a}\right]<1$, we can also have

$$
\begin{aligned}
\alpha_{b, 2 \mid a, 1 \cap \overline{2}} & =P\left[x_{2} \in E_{b} \mid x_{2} \in \overline{E_{a}}, x_{1} \in E_{a}\right] \\
& =P\left[x_{2} \in E_{b} \mid x_{2} \in \overline{E_{a}}\right] \\
& =\frac{P\left[x_{2} \in E_{b}\right]-\alpha_{b \mid a, 2} \cdot P\left[x_{2} \in E_{a}\right]}{1-P\left[x_{2} \in E_{a}\right]} .
\end{aligned}
$$

The reliability of $D M D I_{a, 1 \cap b, 2}$ expressed in (1) can be rewritten by two diversity-related parameters $\alpha_{b \mid a, 2}$ and $\beta_{a, 2 \mid 1}$ as

$$
\begin{aligned}
& R_{D M D I} I_{a, 1 \cap b, 2}=1-P\left[x_{1} \in E_{a}\right] . \\
& {\left[\alpha_{b \mid a, 2} \cdot \beta_{a, 2 \mid 1}+\frac{P\left[x_{2} \in E_{b}\right]-\alpha_{b \mid a, 2} P\left[x_{2} \in E_{a}\right]}{1-P\left[x_{2} \in E_{a}\right]}\left(1-\beta_{a, 2 \mid 1}\right)\right]} \\
& =1-\frac{P\left[x_{1} \in E_{a}\right]}{1-P\left[x_{2} \in E_{a}\right]} \cdot \\
& {\left[\alpha_{b \mid a, 2} \cdot\left(\beta_{a, 2 \mid 1}-P\left[x_{2} \in E_{a}\right]\right)+P\left[x_{2} \in E_{b}\right] \cdot\left(1-\beta_{a, 2 \mid 1}\right)\right] .}
\end{aligned}
$$

Since the reliabilities of $D M S I_{a \cap b, 2}$ and $S M D I_{a, 1 \cap 2}$ can also be expressed using these parameters

$$
\begin{aligned}
& R_{D_{M S I_{a \cap b, 2}}}=1-\alpha_{b \mid a, 2} \cdot P\left[x_{2} \in E_{a}\right], \\
& R_{S I_{a, 1 \cap 2}}=1-\beta_{a, 2 \mid 1} \cdot P\left[x_{1} \in E_{a}\right],
\end{aligned}
$$

the reliabilities of these architectures can be directly compared under given individual modules' reliabilities with two additional variables $\alpha_{b \mid a, 2}$ and $\beta_{a, 2 \mid 1}$. This model corresponds to the previous model presented in [2]. From (16) and (17), we obtain the next proposition.

Proposition 5. Assume that the intersection of errors is conditionally independent of the conjunction of errors, i.e., $\alpha_{b, 2 \mid a, 1 \cap 2}=\alpha_{b \mid a, 2}$ and $\alpha_{b, 2 \mid a, 1 \cap \overline{2}}=P\left[x_{2} \in E_{b} \mid x_{2} \in \overline{E_{a}}\right]$. Given the modules' reliabilities $P\left[x_{i} \in E_{j}\right] \in(0,1), i=$ $\{1,2\}, j=\{a, b\}$, the most reliable architectures among $D M D I_{a, 1 \cap b, 2}, D M S I_{a \cap b, 2}$ and $S M D I_{a, 1 \cap 2}$ can be determined by the following conditions on the values of $\alpha_{b \mid a, 2}$ and $\beta_{a, 2 \mid 1}$.

$$
\left\{\begin{array}{c}
D M S I_{a \cap b, 2}, \quad \text { if } \omega\left(\alpha_{b \mid a, 2}, \beta_{a, 2 \mid 1}\right)-\alpha_{b \mid a, 2} \cdot P\left[x_{2} \in E_{a}\right] \geq 0 \text { and } \\
\beta_{a, 2 \mid 1} \geq \alpha_{b \mid a, 2} \cdot \frac{P\left[x_{2} \in E_{b}\right]}{P\left[x_{1} \in E_{a}\right]}, \\
S M D I_{b, 1 \cap 2}, \quad \text { if } \omega\left(\alpha_{b \mid a, 2}, \beta_{a, 2 \mid 1}\right)-\beta_{a, 2 \mid 1} \cdot P\left[x_{1} \in E_{a}\right] \geq 0 \text { and } \\
\beta_{a, 2 \mid 1} \leq \alpha_{b \mid a, 2} \cdot \frac{P\left[x_{2} \in E_{b}\right]}{P\left[x_{1} \in E_{a}\right]}, \\
\text { otherwise. }
\end{array}\right.
$$

where

$\omega\left(\alpha_{b \mid a, 2}, \beta_{a, 2 \mid 1}\right)=\frac{P\left[x_{1} \in E_{a}\right]}{1-P\left[x_{2} \in E_{a}\right]}$.

$\left[\alpha_{b \mid a, 2} \cdot\left(\beta_{a, 2 \mid 1}-P\left[x_{2} \in E_{a}\right]\right)+P\left[x_{2} \in E_{b}\right] \cdot\left(1-\beta_{a, 2 \mid 1}\right)\right]$. 
Figure 6 visualizes the reliability differences of $D M D I_{a, 1 \cap b, 2}$, $D M S I_{a \cap b, 2}$ and $S M D I_{a, 1 \cap 2}$ by varying the parameter values $\alpha_{b \mid a, 2}$ and $\beta_{a, 2 \mid 1}$ under given modules' probabilities $P\left[x_{1} \in\right.$ $\left.E_{a}\right]=0.1, P\left[x_{2} \in E_{a}\right]=0.2$ and $P\left[x_{2} \in E_{b}\right]=0.3$. The difference of architecture's reliabilities is quantitatively comparable when we obtain the estimates of diversity measures as well as modules' error probabilities.

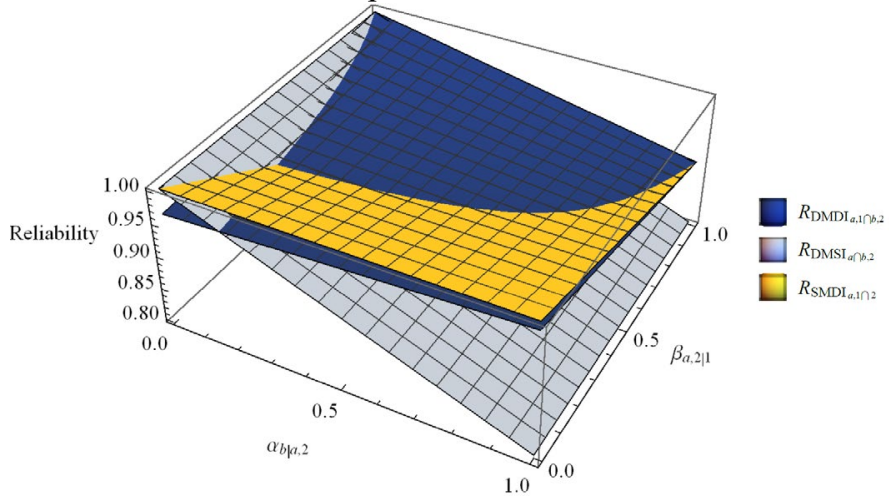

Figure 6. Reliability differences of $D M D I_{a, 1 \cap b, 2}, D M S I_{a \cap b, 2}$ and $S M D I_{a, 1 \cap 2}$

In a similar way, the reliabilities of $D M D I_{a, 2 \cap b, 1}, D M S I_{a \cap b, 1}$ and $S M D I_{b, 1 \cap 2}$ can be directly compared by using the two variables $\alpha_{b \mid a, 1}$ and $\beta_{b, 2 \mid 1}$. However, to compare all the six architectures, we need at least four diversity-associated variables, such as $\left(\alpha_{b \mid a, 1}, \alpha_{b \mid a, 2}, \beta_{a, 2 \mid 1}, \beta_{b, 2 \mid 1}\right)$.

\section{NUMERICAL EXPERIMENTS}

In this section, we present the results of numerical experiments to show the reliabilities of dependent doublemodules double-inputs MLS under hypothetical distribution and error functions. We examine that the properties derived from our reliability model presented in the previous section give the guides to choose the relevant architecture options without having the complete knowledge of input data distribution and error functions.

\section{A. Hypothetical setting}

For the purpose of the experiments, we extend the domain of the joint distribution $\mu_{X_{1}, X_{2}}\left(x_{1}, x_{2}\right)$ to $\mathbb{R}^{2}$. The two continuous random variables $X_{1}$ and $X_{2}$ are assumed to follow $\mu_{X_{1}, X_{2}}\left(x_{1}, x_{2}\right)$. We adopt a bivariate normal distribution

$$
\mu_{X_{1}, X_{2}}\left(x_{1}, x_{2}\right)=\frac{1}{2 \pi \sigma_{X_{1}} \sigma_{X_{2}} \sqrt{1-\rho^{2}}} \cdot \exp \left\{-\frac{z}{2\left(1-\rho^{2}\right)}\right\},
$$

where

$$
z=\frac{\left(x_{1}-\lambda_{X_{1}}\right)^{2}}{\sigma_{X_{1}}^{2}}+\frac{\left(x_{2}-\lambda_{X_{2}}\right)^{2}}{\sigma_{X_{2}}^{2}}-\frac{2 \rho\left(x_{1}-\lambda_{X_{1}}\right)\left(x_{2}-\lambda_{X_{2}}\right)}{\sigma_{X_{1}} \sigma_{X_{2}}},
$$

$\lambda_{X_{1}}$ and $\lambda_{X_{2}}$ are the means, $\sigma_{X_{1}}$ and $\sigma_{X_{2}}$ are the variances, and $\rho$ is the correlation coefficient of $X_{1}$ and $X_{2}$. The dependence of two input data $x_{1}$ and $x_{2}$ can be characterized by the value of $\rho$. The marginal distributions are given by $X_{1} \sim$ $\mathcal{N}\left(\lambda_{X_{1}}, \sigma_{X_{1}}^{2}\right)$ and $X_{2} \sim \mathcal{N}\left(\lambda_{X_{2}}, \sigma_{X_{2}}^{2}\right)$, where $\mathcal{N}\left(\lambda, \sigma^{2}\right)$ represents the normal distribution with mean $\lambda$ and variance $\sigma^{2}$. For the sake of simplicity, we define error input spaces for individual ML models $m_{a}$ and $m_{b}$ by closed intervals on $\mathbb{R}$ as
$E_{a}=\left[e_{a}^{\min }, e_{a}^{\max }\right]$ and $E_{b}=\left[e_{b}^{\min }, e_{b}^{\max }\right]$. With this hypothetical setting, an example relation between two different error spaces with a joint distribution of two input data can be visualized as shown in Figure 7, where the horizontal and vertical axes represent the values of $x_{1}$ and $x_{2}$, respectively.

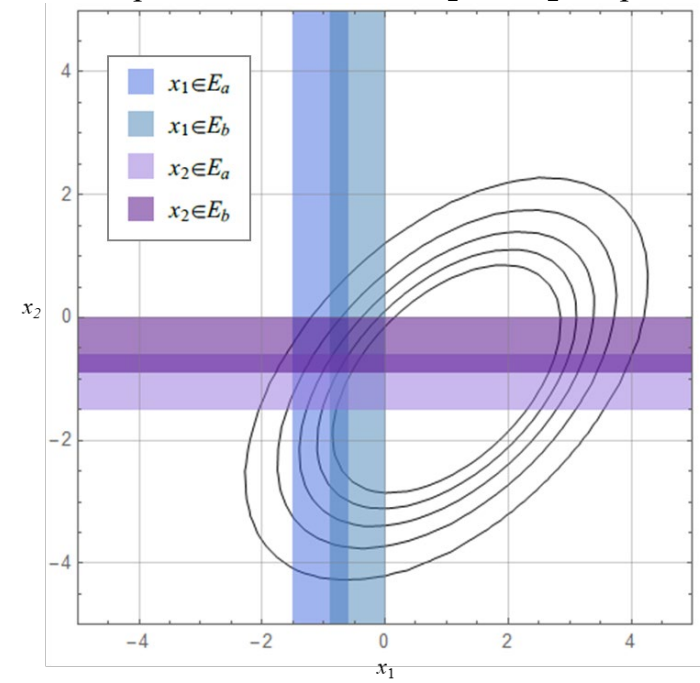

Figure 7. Error input spaces over binormal distribution with $\rho=0.5$

The colored regions show the error spaces for $x_{1} \in$ $[-1.5,0.6], x_{1} \in[-0.9,0], x_{2} \in[-1.5,0.6]$ and $x_{2} \in[-0.9,0]$, while the circles represent the counter lines shaped by the bivariate normal distribution with the parameters $\left(\lambda_{X_{1}}, \lambda_{X_{2}}, \sigma_{X_{1}}^{2}, \sigma_{X_{2}}^{2}, \rho\right)=(1,-1,1.5,1.5,0.5)$. Though the hypothetical distribution and error spaces are not real, such a visualization gives an intuitive view of the interrelation between dependent input data and dependent error spaces. For example, consider the point $\left(x_{1}, x_{2}\right)=(-0.1,2)$, ML model $m_{b}$ outputs error for $x_{1}$, but neither model outputs error for $x_{2}$. The probability density of such a case can be given by $\mu_{X_{1}, X_{2}}(-0.1,2)$. The probability of error output by an ML module can be computed by integrating the probability density of the corresponding input data and the error space. For example, the probability of $x_{1} \in E_{a}$ is given by

$$
\begin{aligned}
P\left[x_{1} \in E_{a}\right] & =\int f_{a}\left(x_{1}\right) d \mu_{X_{1}, X_{2}}\left(x_{1}, x_{2}\right)=\int f_{a}\left(x_{1}\right) d \mu_{X_{1}}\left(x_{1}\right) \\
& =\int_{e_{a}^{\min }}^{e_{a}^{\max }} \mu_{X_{1}}\left(x_{1}\right) d x_{1} .
\end{aligned}
$$

The reliabilities of $D M S I_{a \cap b, 1}, S M D I_{a, 1 \cap 2}$ and $D M D I_{a, 1 \cap b, 2}$ can be computed by

$$
\begin{aligned}
& R_{\text {DMSI }_{a n b, 1}}=1-\int f_{a}\left(x_{1}\right) f_{b}\left(x_{1}\right) d \mu_{X_{1}}\left(x_{1}\right) \\
& =\left\{\begin{array}{cc}
\int_{\max \left(e_{a}^{\min }, e_{b}^{\min }\right)}^{\min \left(e_{a}^{\max }, e_{b}^{\max }\right)} \mu_{X_{1}}\left(x_{1}\right) d x_{1}, & \begin{array}{c}
\max \left(e_{a}^{\min }, e_{b}^{\min }\right)< \\
\min \left(e_{a}^{\max }, e_{b}^{\max }\right)
\end{array} \\
0, & \text { otherwise, }
\end{array}\right. \\
& R_{S M D I_{a, 1 \cap 2}}=1-\int f_{a}\left(x_{1}\right) f_{a}\left(x_{2}\right) d \mu_{X_{1}, X_{2}}\left(x_{1}, x_{2}\right) \\
& =\int f_{a}\left(x_{1}\right)\left[\int_{e_{a}^{\min }}^{e_{a}^{\max }} \mu_{X_{1}, X_{2}}\left(x_{1}, x_{2}\right) d x_{2}\right] d \mu_{X_{1}}\left(x_{1}\right)
\end{aligned}
$$




$$
\begin{aligned}
= & \int_{e_{a}^{\min }}^{e_{a}^{\max }} \int_{e_{a}^{\min }}^{e_{a}^{\max }} \mu_{X_{1}, X_{2}}\left(x_{1}, x_{2}\right) d x_{2} d x_{1}, \\
R_{D M D I_{a, 1 \cap b, 2}} & =1-\int_{a} f_{a}\left(x_{1}\right) f_{b}\left(x_{2}\right) d \mu_{X_{1}, X_{2}}\left(x_{1}, x_{2}\right) \\
& =\int_{e_{a}^{\min }}^{e_{a}^{\max }} \int_{e_{b}^{\min }}^{e_{b}^{\max }} \mu_{X_{1}, X_{2}}\left(x_{1}, x_{2}\right) d x_{2} d x_{1} .
\end{aligned}
$$

The reliabilities of $D M S I_{a \cap b, 2}, S M D I_{b, 1 \cap 2}$ and $D M D I_{a, 2 \cap b, 1}$ can also be computed in a similar way.

\section{B. Architecture comparison by varying input similarity}

Using the abovementioned hypothetical setting, we numerically investigate the impact of dependency of two input data on the architecture reliabilities. The dependency of two input data distributions resulting from $\mu_{X_{1}, X_{2}}\left(x_{1}, x_{2}\right)$ can be characterized by the correlation coefficient $\rho$. When $\rho=0$, the two input data $X_{1}$ and $X_{2}$ are independent of each other. On the other hand, when $|\rho|$ is close to $1, X_{1}$ has a strong positive or negative correlation with $X_{2}$. We set $E_{a}=[-0.4,0.1], E_{b}=$ $[0,0.5]$ and $\left(\lambda_{X_{1}}, \lambda_{X_{2}}, \sigma_{X_{1}}^{2}, \sigma_{X_{2}}^{2}\right)=(1,-1,1.5,1.5)$ and vary the value of $\rho$ in the range of $[-0.9,0.9]$.

The computed reliabilities of the six architectures are shown in Figure 8. As can be seen, the reliabilities of DMDI and SMDI architectures are affected by changing the values of $\rho$, while the reliabilities of DMSI architectures are not affected. The results make sense because DMSI architecture does not use multiple input data, and hence the correlation of different input data does not influence their reliabilities. It is interesting that DMSI architectures achieve the highest reliabilities when $\rho$ is close to -1 among six architectures while they become the least reliabilities when $\rho>-0.7$. In most of the range, $D M D I_{a, 1 \cap b, 2}$ is considered as the best architecture in terms of reliability.

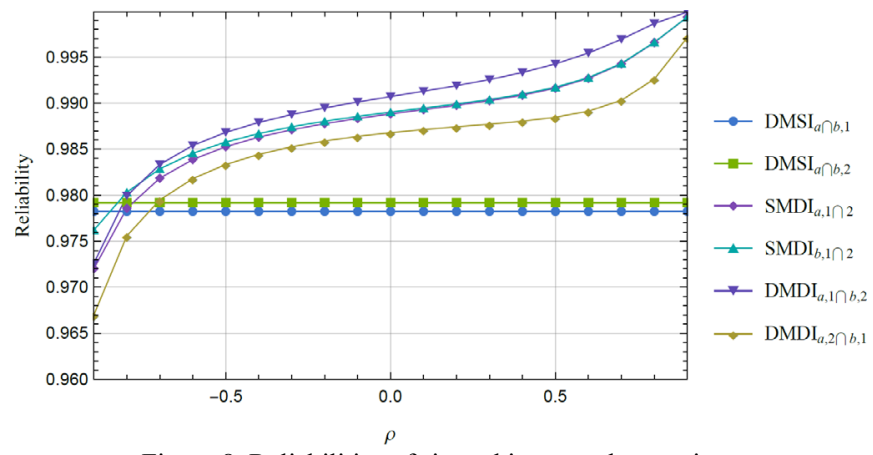

Figure 8. Reliabilities of six architectures by varying $\rho$

In practice, we do not know the exact distribution $\mu_{X_{1}, X_{2}}\left(x_{1}, x_{2}\right)$ or error input spaces $E_{a}$ and $E_{b}$. The comparative results of architecture reliabilities shown in Figure 8 are not obtainable from the real observation. However, the preferable architectures can be inferred partly from Proposition 4 , since under the given distribution and the error spaces, we can expect $P\left[x_{1} \in E_{a}\right] \leq P\left[x_{2} \in E_{a}\right]$ and $P\left[x_{2} \in E_{b}\right] \leq$ $P\left[x_{1} \in E_{b}\right]$ are likely hold. When we observe that the above relations are generally held in the target application, by Proposition 4, either $D M D I_{a, 1 \cap b, 2}, S M D I_{a, 1 \cap 2}$ or $S M D I_{b, 1 \cap 2}$ can be selected as the preferable architectures.
We investigate the influence of the conjunction of errors $\beta_{a, 2 \mid 1}$ and $\beta_{b, 2 \mid 1}$ in this experimental setting. The value of $\rho$ is associated with the values of $\beta_{a, 2 \mid 1}$ and $\beta_{b, 2 \mid 1}$. When the error spaces and input data distribution are given, the diversity measure can be computed by

$$
\begin{aligned}
\beta_{j, 2 \mid 1} & =\operatorname{Pr}\left[x_{2} \in E_{j} \mid x_{1} \in E_{j}\right] \\
& =\frac{\int_{e_{j}^{\min }}^{e_{j}^{\max }} \int_{e_{j}^{\min }}^{e_{j}^{\max }} \mu_{X_{1}, X_{2}}\left(x_{1}, x_{2}\right) d x_{2} d x_{1}}{\int_{e_{j}^{\min }}^{e_{\max }^{\max }} \mu_{X_{1}}\left(x_{1}\right) d x_{1}}, j=a, b .
\end{aligned}
$$

By varying the value of $\rho$, the values of $\beta_{a, 2 \mid 1}$ and $\beta_{b, 2 \mid 1}$ change as plotted in Figure 9 .

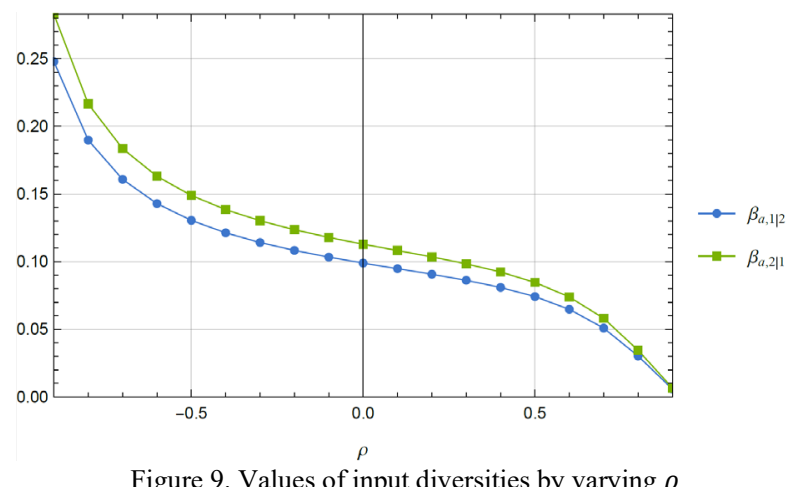

As can be seen, there are negative correlations between them. The higher $\rho$ leads to the smaller $\beta_{a, 2 \mid 1}$ and $\beta_{a, 1 \mid 2}$, resulting in the higher reliabilities of DMDI and SMDI architectures. From Proposition 1, the conditions where the reliability of $S M D I_{a, 1 \cap 2}$ becomes lower than the reliabilities of DMSI and DMDI architectures are given by $\boldsymbol{B}_{a}^{+\top}>\boldsymbol{A}_{b \mid a} \cdot \boldsymbol{B}_{a}^{\top}$. For $D M S I_{a \cap b, 1}$ and $D M D I_{a, 1 \cap b, 2}$, the conditions on $\beta_{a, 2 \mid 1}$ are given by;

$$
\left\{\begin{array}{l}
R_{S M D I_{a, 1 \cap 2}}<R_{D M S I_{a \cap b, 1}} \Leftrightarrow \\
\beta_{a, 2 \mid 1}>\frac{\alpha_{b, 1 \mid a, 1 \cap \overline{2}}}{1-\alpha_{b, 1 \mid a, 1 \cap 2}+\alpha_{b, 1 \mid a, 1 \cap \overline{2}}}\left(=D M S I_{a \cap b, 1} \text { bound }\right), \\
R_{S M D I_{a, 1 \cap 2}}<R_{D M D I_{a, 1 \cap b, 2}} \Leftrightarrow \\
\beta_{a, 2 \mid 1}>\frac{\alpha_{b, 2 \mid a, 1 \cap \overline{2}}}{1-\alpha_{b, 2 \mid a, 1 \cap 2}+\alpha_{b, 2 \mid a, 1 \cap \overline{2}}}\left(=D M D I_{a, 1 \cap b, 2} \text { bound }\right) .
\end{array}\right.
$$

Figure 10 plots the values of the above bounds together with $\beta_{a, 2 \mid 1}$ by varying $\rho$.

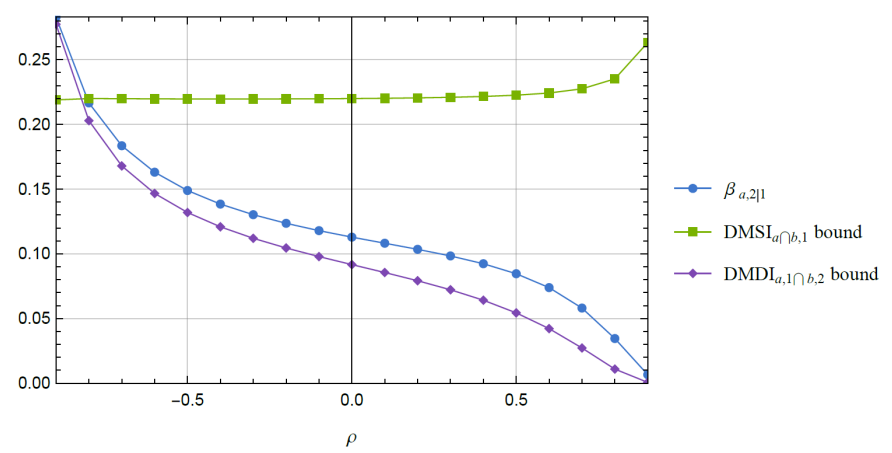

Figure 10. Bounds of $\beta_{a, 2 \mid 1}$ for DMSI and DMDI architectures 
$\beta_{a, 2 \mid 1}$ is constantly larger than $D M D I_{a, 1 \cap b, 2}$ bound regardless of $\rho$, which means $D M D I_{a, 1 \cap b, 2}$ is always a better choice than $S M D I_{a, 1 \cap 2}$. On the other hand, $\beta_{a, 2 \mid 1}$ is smaller than $D M S I_{a \cap b, 1}$ bound in most of the range of $\rho(\geq-0.8)$, which indicates that $S M D I_{a, 1 \cap 2}$ is the better choice than $D M S I_{a \cap b, 1}$ unless $\beta_{a, 2 \mid 1}$ tends to be large.

\section{Architecture comparison by varying model similarity}

Next, we investigate the effects of dependency between two ML models that have different error spaces. In this experiment, we fix the parameters of bivariate normal distribution $\left(\lambda_{X_{1}}, \lambda_{X_{2}}, \sigma_{X_{1}}^{2}, \sigma_{X_{2}}^{2}, \rho\right)=(1,-1,1.5,1.5,0.5)$ and the error space $E_{b}=[0,0.5]$. The other error space is given by $E_{a}=$ $\left[e_{a}^{\min }, e_{a}^{\min }+0.5\right]$ where $e_{a}^{\min }$ is varying in the range of [$0.5,0]$. Figure 11 shows $E_{a}$ and $E_{b}$ in the interval [-0.5, 0.5].

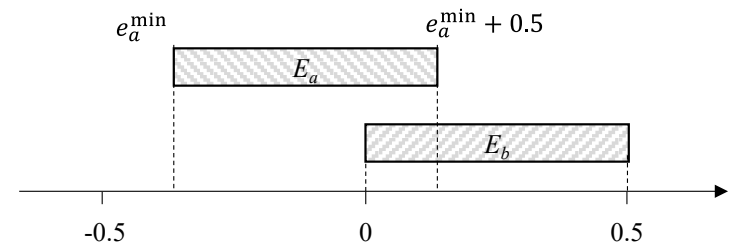

Figure 11. The intersection of errors between $\boldsymbol{E}_{\boldsymbol{a}}$ and $\boldsymbol{E}_{\boldsymbol{b}}$

When $e_{a}^{\min }=-0.5$, there is no intersection between $E_{a}$ and $E_{b}$, and thus the value of model similarity is equal to zero. On the other hand, when $e_{a}^{\min }=0, E_{a}$ becomes identical to $E_{b}$ and hence the value of model similarity becomes one. Figure 12 shows the reliabilities of six different architecture by varying the value of $e_{a}^{\mathrm{min}}$.

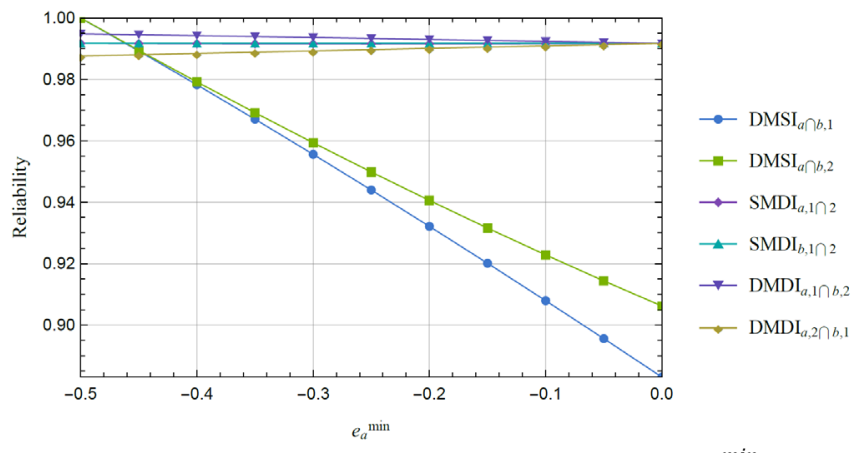

Figure 12. Reliabilities of six architectures by varying $e_{a}^{\min }$

As can be seen, the reliabilities of both DMSI architectures decrease by increasing the values of $e_{a}^{\mathrm{min}}$, while the reliabilities of DMSI and DMDI architectures are not much affected. Since the intersection of error spaces between $E_{a}$ and $E_{b}$ increases by the increase in $e_{a}^{\min }$, the error probabilities of DMSI architectures also increase accordingly. When $e_{a}^{\min }$ is close to -0.5 , the intersection over $E_{b}$ becomes extremely small, and then DMSI architectures achieve the highest reliabilities. In most of the range, however, $D M D I_{a, 1 \cap b, 2}$ achieves the highest reliability among the six architectures. Regardless of the position of $E_{a}$, the condition of Proposition 4 is likely to hold. Thus, by Proposition 4 , we can infer that $D M D I_{a, 1 \cap b, 2}$ or SMDI architectures are preferable even we do not know the exact distribution of input data or the error input spaces.

We investigate the influence of the intersection of errors $\alpha_{b \mid a, 1}$ and $\alpha_{b \mid a, 2}$ in this experimental setting. For the given input data distributions and the error spaces $E_{b}=[0,0.5]$ and $E_{a}=\left[e_{a}^{\min }, e_{a}^{\min }+0.5\right]$ where $e_{a}^{\min }=[-0.5,0]$, the diversity measures can be computed by

$$
\begin{aligned}
& \alpha_{b \mid a, i}=\operatorname{Pr}\left[x_{i} \in E_{b} \mid x_{i} \in E_{a}\right] \\
& =\frac{\int_{e_{b}^{\min }}^{e_{a}^{\min }+0.5} \mu_{X_{i}}\left(x_{i}\right) d x_{i}}{\int_{e_{a}^{\min }}^{e_{\min }^{\min }+0.5} \mu_{X_{i}}\left(x_{i}\right) d x_{i}}, \quad i=1,2 .
\end{aligned}
$$

By varying the value of $e_{a}^{\min }$, the values of $\alpha_{b \mid a, 1}$ and $\alpha_{b \mid a, 2}$ change as shown in Figure 13.

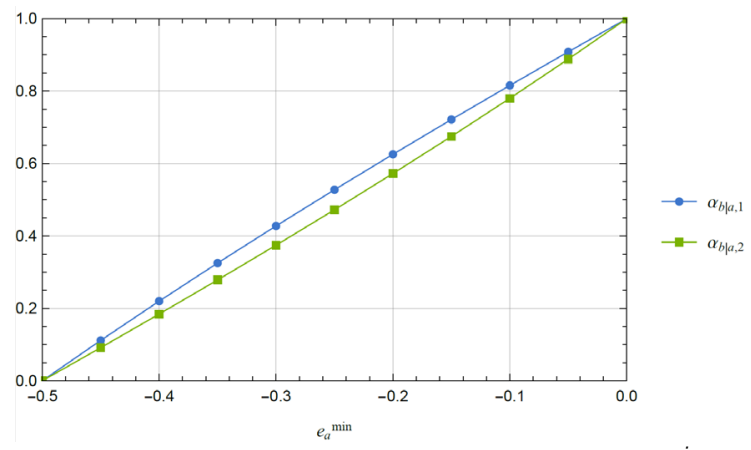

Figure 13. Values of model diversities by varying $e_{a}^{\min }$

As expected, we can observe both $\alpha_{b \mid a, 1}$ and $\alpha_{b \mid a, 2}$ have positive correlations with $e_{a}^{\min }$, resulting in the lower reliabilities of DMSI architectures. Therefore, when we observe the larger values of $\alpha_{b \mid a, 1}$ or $\alpha_{b \mid a, 2}$, by the variant of Proposition 1, DMSI architectures are unlikely the appropriate architecture options in terms of reliability.

\section{Special distribution case}

For an instance of the restricted type of distribution discussed in Section IV-B, next we consider the case where errors in $E_{b}$ occur more frequently than errors in $E_{a}$, and examine Proposition 3. Let consider the bivariate normal distribution with the parameters $\left(\lambda_{X_{1}}, \lambda_{X_{2}}, \sigma_{X_{1}}^{2}, \sigma_{X_{2}}^{2}\right)=(0,0,1.5,1.5)$. Regardless of the value of correlation coefficient $\rho$, the marginal distribution is given by $\mathcal{N}(0,1.5)$. When we fix the error spaces $E_{a}=[-1.4,-0.9]$ and $E_{b}=[-1.0,-0.5]$, it satisfies $P\left[x_{i} \in E_{a}\right] \leq P\left[x_{i} \in E_{b}\right]$ for most of input data $x_{i}$ that follows the input data distribution $\mathcal{N}(0,1.5)$. The intuition of this relation can be given from Figure 14, as we can see that the region of $E_{b}$ is always closer to the peak of probability density at (0.0) than the region of $E_{a}$.

From Proposition 3, the most reliable architecture is either $S M D I_{a, 1 \cap 2}, D M S I_{a \cap b, 1}$ or $D M S I_{a \cap b, 2}$ depending on the values of $\alpha_{b \mid a, 1}$ and $\alpha_{b \mid a, 2}$. Since the given bivariate normal distribution has symmetry in marginal distributions, the condition can be simplified as

$$
\left\{\begin{array}{l}
D M S I_{a \cap b, 1}, \\
S_{b M \mid a, 1} \leq \frac{\beta_{a, 2 \mid a \cap \bar{b}, 1}}{1-\beta_{a, 2 \mid a \cap b, 1}+\beta_{a, 2 \mid a \cap \bar{b}, 1}}, \\
\text { otherwise. }
\end{array}\right.
$$




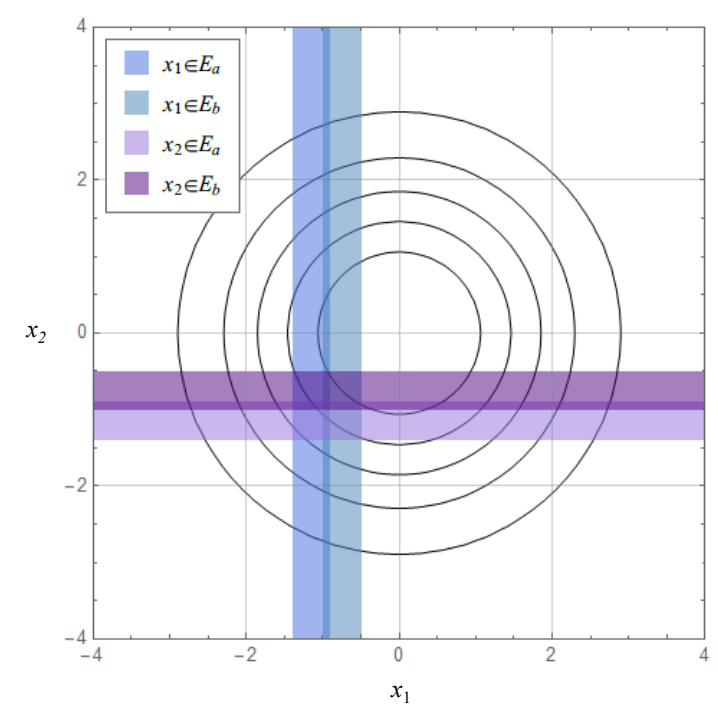

Figure 14. An example of error spaces satisfying $P\left[x_{i} \in E_{a}\right] \leq P\left[x_{i} \in E_{b}\right]$

Figure 15 shows the architecture reliabilities directly computed from the given distribution by varying $\rho$ in the rage of $[-0.9,0.9]$. Due to the symmetry marginal distributions, we can observe $R_{D M S I_{a \cap b, 1}}=R_{D M S I_{a \cap b, 2}}$ and $R_{D M D I_{a, 1 \cap b, 2}}=$ $R_{D M D I_{a, 2 n b, 1}}$. In the most of range of $\rho, S M D I_{a, 1 \cap 2}$ achieves the highest reliability.

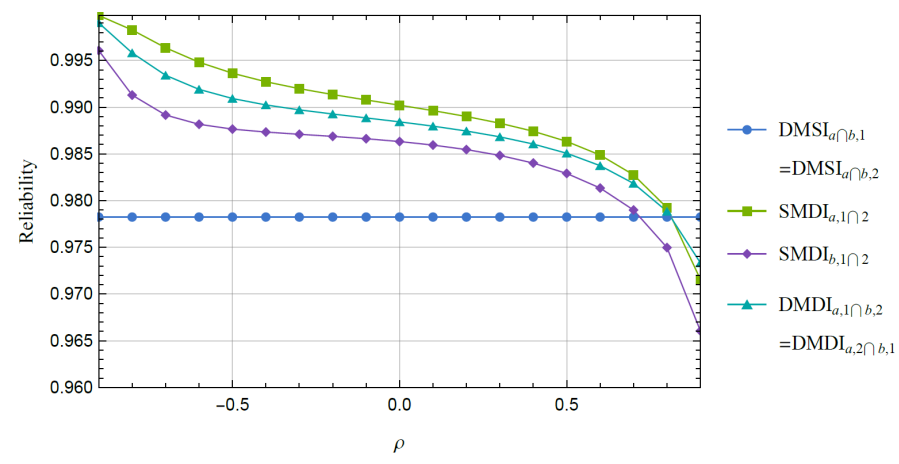

Figure 15. Reliabilities of different architectures by varying $\rho$

Figure 16 plots the $\alpha_{b \mid a, 1}$ and the boundary condition given by Proposition 3.

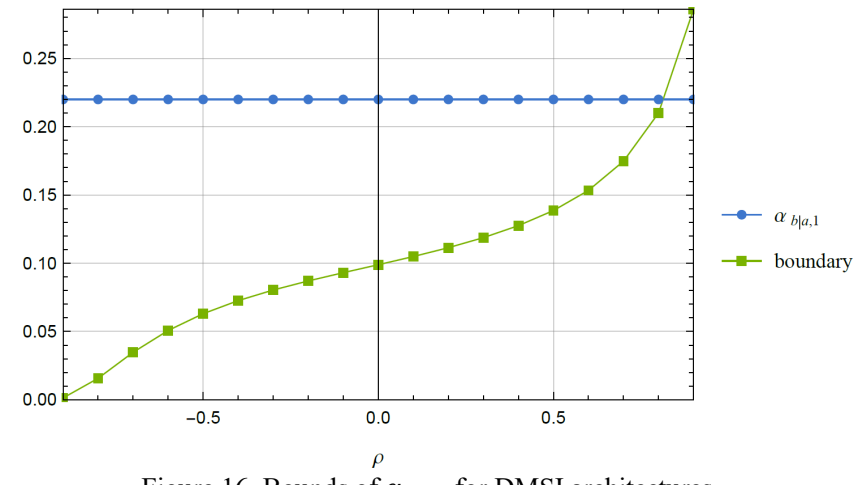

Figure 16. Bounds of $\alpha_{b \mid a, 1}$ for DMSI architectures

The value of $\alpha_{b \mid a, 1}$ becomes lower than the boundary when $\rho=0.9$ at which $D M S I_{a \cap b, 1}$ achieves higher reliability than
$S M D I_{a, 1 \cap 2}$ as observed in Figure 15. The result confirms the condition of Proposition 3. Unless we are confident that $\alpha_{b \mid a, 1}$ is very small value from empirical observation, $S M D I_{a, 1 \cap 2}$ can be chosen as the preferable architecture option.

\section{E. Limitations}

The results of numerical experiments show that our reliability model and the derived properties provide the guides to choose the preferable architecture options based on the diversity measures. However, without knowing the exact values of diversity measures, it is impossible to determine the best architecture option. In practice, we may need to rely on empirical estimates of the diversity measures from the limited samples, which may contain estimation errors as well. Instead of deciding the best architecture options, our reliability model can be used to select the preferable architectures by screening undesirable architecture options. In future studies, it is an important challenge to provide the method or framework to select the preferable architecture options through a specific experimental procedure to obtain the estimations of diversity measures.

\section{RELATED WORK}

Quality assurance of MLS has recently become a hot issue in software and system engineering research. MLS is fundamentally built as a software system, and therefore developers of MLS apply and improve the traditional software engineering methodologies to assure the quality of $\mathrm{ML}$ application systems [14]. In this regard, one of the important challenges actively discussed today is machine learning testing [10]. Compared to traditional software testing, MLS testing needs to deal with an oracle problem [19]. What the correct output should be for arbitrary input is not given when testing an MLS. The software systems which have no reliable test oracle are known as non-testable programs [20]. To address the oracle issue of MLS, Murphy, and Kaiser [21] presented a metamorphic testing approach in which pseudo-oracle [22] for new test cases is created by modifying the inputs used in the initial test cases. Metamorphic testing has been recently applied for testing image classifiers [15], deep learning-based forecaster [16], and graph convolutional neural networks [17]. Further recent advances in ML testing techniques are reviewed in a comprehensive survey report [23]. Our study is not a kind of MLS testing method, but the presented architecture reliability analysis can be complementary for developing highly reliable MLSs.

Another important technical challenge in MLS quality management is the runtime validation of MLS execution. Even if an MLS passes reasonably designed test scenarios, the outputs of MLS are significantly influenced by real input data given in users' environments. The discrepancy between the assumed input data in the development phase and the real input data observed in the operation phase likely causes undesirable outputs at runtime. $\mathrm{Wu}$ et al. leveraged the data validation technique to detect real-world corner cases for DNN-based systems [24]. Considering a cyber-physical system employs ML components, Dreossi et al. proposed a method to analyze 
the input space for ML classifiers that can lead to undesirable consequences in the cyber-physical system [25]. We also take into account the operational phase of MLS, while the purpose of the study is not validation but evaluation of reliability by Nversion MLS.

Redundant configuration of ML components can improve the reliability of MLS. N-version machine learning has been presented to improve the reliability of MLS's outputs [2]. The relationship between the reliability of N-version MLS and the diversities in ML models and input data has been analyzed in our previous study [2]. The experimental studies also showed that the coverage of errors could be improved by using diverse ML models and different input data sets [6]. Similar experiments have been done by $\mathrm{Xu}$ et al. in which $\mathrm{N}$-version deep neural networks are used to complement individual error cases and make a fault-tolerant system [5]. A Multi-version approach has also been used for ML applications using deep neural networks to make more robust decisions of steering control in an autonomous vehicle [26]. PolygraphMR also introduced modular redundancy to reduce the high confidence wrong answers from the system [18]. In this paper, extending the diversity measures introduced in [2], we present more comprehensive and general reliability models for MLS. To the best of our knowledge, our study is the first work to show the reliability properties of dependent double-modules doubleinputs MLS via diversity measures.

Using multiple learners to compose a better ML model is a commonly adopted ML technique that is also known as the ensemble method [27]. To obtain a better ensemble, individual learners should have differences among them. It is studied that the minimum margin of the ensemble is associated with the diversity among the individual learners [28]. A number of diversity measures were proposed to analyze the association with the accuracy of ensemble methods [29][30][31][32]. Although diversity in general, is believed to play a fundamental role in ensemble accuracy, the right formulation and measures for diversity are unsolved issues [33]. Empirical studies show that diverse ML models can be constructed by using the different data sets, sampling methods, training algorithms, and neural network architectures [5][6][34]. In this paper, we focus on the reliability of MLS outputs that is directly related to the accuracies of individual ML models used in the system. While highly accurate ML models likely improve the reliability of MLS, it is valid only when real input data distributions follow the assumption or expectation. Instead of the accuracy of the ML model, we discuss the reliability of MLS attributed by redundant configuration that consists of different input data and ML models. Since both input data sets and ML models are not independent of each other, we cannot formulate the problem by a simple combinatorial logic that is the challenge addressed in this paper.

\section{CONCLUSION}

This paper proposed the reliability model for an N-version MLS that employs two ML modules for classification tasks and determines the system output on a consensus basis. The system has six architecture options depending on the choice of input data and ML models used in the modules. We defined two diversity measures to quantify the similarities of ML models' capabilities and the interdependence of input data sets. The diversity measures are used to formulate the reliabilities of six different architectures. With the proposed matrix representation of the reliability model, we showed some important properties that guide the choice of preferable architecture when given individual ML models' reliabilities and diversity measures. Among possible architectures, DMDI architecture, which exploits both model diversity and input diversity, can be regarded as a neutral and a conservative option since the values of diversity measures are unlikely to be close to zero or one in practice. We also presented the numerical experimental results to give an intuitive understanding of our proposed models and the derived properties. In practice, it is still a big challenge to infer the diversity parameters used in our model. However, our study first contributes to clarifying the relationship between the reliability of MLS with redundancy and the inherent dependencies in diverse input data as well as in ML models.

Extending the reliability model to multi-modules multiinputs MLS could be explored in a future study. Although our study is initially motivated by N-version MLS, this paper focuses on a dependent double-modules double-inputs MLS that could be a fundamental building block of any larger Nversion system. Another important future research direction is the validation of the models with real-world data set. Our previous work also presented some experimental results that show two potential ways to diversify the outputs of redundant MLS [6]. Because the input data distributions and error spaces are hard to obtain empirically, future studies are required to narrow the gap between the limited empirical results and the theoretically derived properties.

\section{APPENDIX}

Proof of Lemma 1. Consider the reliability difference between $D M S I_{a \cap b, 1}$ and $S M D I_{a, 1 \cap 2}$, from the expression (10), the first-row first-column element of $\boldsymbol{H}_{b \mid a}$ is

$$
\begin{gathered}
{\left[\beta_{a, 2 \mid 1}-\left\{\alpha_{b, 1 \mid a, 1 \cap 2} \cdot \beta_{a, 2 \mid 1}+\alpha_{b, 1 \mid a, 1 \cap \overline{2}} \cdot\left(1-\beta_{a, 2 \mid 1}\right)\right\}\right]} \\
\cdot P\left[x_{1} \in E_{a}\right] \\
=\left\{\left(1-\alpha_{b, 1 \mid a, 1 \cap 2}+\alpha_{b, 1 \mid a, 1 \cap \overline{2}}\right) \cdot \beta_{a, 2 \mid 1}-\alpha_{b, 1 \mid a, 1 \cap \overline{2}}\right\} \\
\cdot P\left[x_{1} \in E_{a}\right] .
\end{gathered}
$$

Since $1-\alpha_{b, 1 \mid a, 1 \cap 2}+\alpha_{b, 1 \mid a, 1 \cap \overline{2}}>0$, the difference becomes larger as $\beta_{a, 2 \mid 1}$ increases. Similarly, the reliability difference between $D M D I_{a, 1 \cap b, 2}$ and $S M D I_{a, 1 \cap 2}$, which corresponds to the second-row first-column element of $\boldsymbol{H}_{b \mid a}$, is shown to be increased when $\beta_{a, 2 \mid 1}$ increases. On the other hand, the reliability differences for $D M D I_{a, 2 \cap b, 1}$ and $D M S I_{a \cap b, 2}$ against $S M D I_{a, 1 \cap 2}$ are shown to be increased when $\beta_{a, 1 \mid 2}$ increases by examining the second-row elements of $\boldsymbol{H}_{b \mid a}$.

Proof of Lemma 2. From Lemma 1 and expression (10), the proof is relatively straightforward. Due to the monotonicity with respect to $\beta_{a, 2 \mid 1}$ and $\beta_{a, 1 \mid 2}$, the lower bound of $\boldsymbol{H}_{b \mid a}$ is given when $\beta_{a, 2 \mid 1}$ and $\beta_{a, 1 \mid 2}$ are at their lower bounds. When there is no possibility of error conjunction in $E_{a}$, the values 
of diversity $\beta_{a, 2 \mid 1}$ and $\beta_{a, 1 \mid 2}$ asymptotically approach to zero. Considering the limiting case of $\boldsymbol{H}_{b \mid a}\left(\beta_{a, 2 \mid 1}, \beta_{a, 1 \mid 2} \rightarrow 0\right)$, we can obtain $\boldsymbol{H}_{b \mid a}^{\min }$. On the other hand, the upper bounds of $\beta_{a, 2 \mid 1}$ and $\beta_{a, 1 \mid 2}$ change depending on the relation of $P\left[x_{1} \in\right.$ $\left.E_{a}\right]$ and $P\left[x_{2} \in E_{a}\right]$ as below

$$
\begin{array}{r}
\sup \beta_{a, 2 \mid 1}=\left\{\begin{array}{rr}
\frac{P\left[x_{2} \in E_{a}\right]}{P\left[x_{1} \in E_{a}\right]}, & P\left[x_{2} \in E_{a}\right]<P\left[x_{1} \in E_{a}\right] \\
1, & P\left[x_{2} \in E_{a}\right] \geq P\left[x_{1} \in E_{a}\right], \\
1, & P\left[x_{2} \in E_{a}\right]<P\left[x_{1} \in E_{a}\right]
\end{array}\right. \\
\sup \beta_{a, 1 \mid 2}=\left\{\begin{array}{rr}
P\left[x_{1} \in E_{a}\right] \\
\frac{P\left[x_{2} \in E_{a}\right]}{}, & P\left[x_{2} \in E_{a}\right] \geq P\left[x_{1} \in E_{a}\right] .
\end{array}\right.
\end{array}
$$

Applying the above upper bounds of $\beta_{a, 2 \mid 1}$ and $\beta_{a, 1 \mid 2}$ to (10) yields $\sup \boldsymbol{H}_{b \mid a}$.

Proof of Proposition 2. We can derive the conditions by examining the signs of the corresponding elements of $\boldsymbol{H}_{b \mid a}$ and $\boldsymbol{H}_{a \mid b}$. From the assumption and Lemma 2, $\sup \boldsymbol{H}_{b \mid a}=$ $\boldsymbol{H}_{b \mid a}^{\text {sup2 }}$. Hence the element of $\sup \boldsymbol{H}_{b \mid a}$ for $D M S I_{a \cap b, 1}$, which is the first-row first-column element, is given by $1-$ $\alpha_{b, 1 \mid a, 1 \cap 2}>0$. By Proposition 1, SMDI $I_{a, 1 \cap 2}$ achieves higher or equal reliability than $D M S I_{a \cap b, 1}$ only when $\boldsymbol{B}_{a}^{+\top} \leq \boldsymbol{A}_{b \mid a}$. $\boldsymbol{B}_{a}^{\top}$ satisfies at the first-row first-column element. Thus, we have the condition

$$
\beta_{a, 2 \mid 1} \leq \frac{\alpha_{b, 1 \mid a, 1 \cap \overline{2}}}{1-\alpha_{b, 1 \mid a, 1 \cap 2}+\alpha_{b, 1 \mid a, 1 \cap \overline{2}}} .
$$

Similarly, from the assumption, $\sup \boldsymbol{H}_{a \mid b}=\boldsymbol{H}_{a \mid b}^{\text {sup2 }}$ and hence the element for $D M S I_{a \cap b, 1}$ of $\sup \boldsymbol{H}_{a \mid b}$ is given by $1-\alpha_{a, 1 \mid b, 1 \cap 2}>0$. Following the dual of Proposition 1, $S M D I_{b, 1 \cap 2}$ achieves higher or equal reliability than $D_{M S I_{a \cap b, 1}}$ only when $\boldsymbol{B}_{b}^{+\top} \leq \boldsymbol{A}_{a \mid b} \cdot \boldsymbol{B}_{b}^{\top}$ satisfies at the firstrow first-column element. Thus, we have the condition

$$
\beta_{b, 2 \mid 1} \leq \frac{\alpha_{a, 1 \mid b, 1 \cap \overline{2}}}{1-\alpha_{a, 1 \mid b, 1 \cap 2}+\alpha_{a, 1 \mid b, 1 \cap \overline{2}}} .
$$

Finally, since $R_{S M D I_{a, 1 \cap 2}}=1-\beta_{a, 2 \mid 1} \cdot P\left[x_{1} \in E_{a}\right]$ and $R_{S M D I_{b, 1 \cap 2}}=1-\beta_{b, 2 \mid 1} \cdot P\left[x_{1} \in E_{b}\right]$, the condition where $S M D I_{a, 1 \cap 2}$ achieves higher or equal reliability than $S M D I_{b, 1 \cap 2}$ is given by

$$
\beta_{a, 2 \mid 1} \leq \beta_{b, 2 \mid 1} \cdot \frac{P\left[x_{1} \in E_{b}\right]}{P\left[x_{1} \in E_{a}\right]} .
$$

From (18)(19)(20), we can derive the conditions for the most reliable architecture.

\section{REFERENCES}

[1] R. McAllister, Y. Gal, A. Kendall, M. van der Wilk, A. Shah, R. Cipolla, A. Weller, Concrete problems for autonomous vehicle safety: advantages of Bayesian deep learning, In Proc. of the 26th International Joint Conference on Artificial Intelligence (IJCAI), pp. 4745-4753, 2017.

[2] F. Machida, N-version machine learning models for safety critical systems, In Proc. of the DSN Workshop on Dependable and Secure Machine Learning, pp. 48-51, 2019.

[3] A. Avizienis and L. Chen, On the implementation of N-version programming for software fault tolerance during execution. In Proc. of IEEE International Computer, Software and Application Conference (COMPSAC), pp. 149-155, 1977.
[4] A. Avizienis, The methodology of n-version programming, Software fault tolerance, Vol. 3, pp. 23-46, John Wiley \& Sons, New York, 1995.

[5] H. Xu, Z. Chen, W. Wu, Z. Jin, S. Kuo, M. R. Lyu, NV-DNN: towards fault-tolerant DNN systems with N-version programming, In Proc. of the Workshop on Dependable and Secure Machine Learning, pp. 44-47, 2019.

[6] F. Machida, On the diversity of machine learning models for system reliability, IEEE Pacific Rim Int'l Symp. on Dependable Computing (PRDC), pp. 276-285, 2019.

[7] K. Pei, Y. Cao, J. Yang, and S. Jana, DeepXplore: Automated whitebox testing of deep learning systems, In Proc. of the 26th Symposium on Operating Systems Principles (SOSP), pp. 1-18, 2017.

[8] Y. Tian, K. Pei, S. Jana, and B. Ray, Deeptest: Automated testing of deep-neural-network-driven autonomous cars, In Proceedings of the 40th international conference on software engineering, pp. 303-314, 2018.

[9] Y. Zhang, Y. Chen, S. C. Cheung, Y. Xiong, and L. Zhang, An empirical study on tensorflow program bugs, In Proceedings of the 27th ACM SIGSOFT International Symposium on Software Testing and Analysis, pp. 129-140, 2018.

[10] H. B. Braiek and F. Khomh, On testing machine learning programs, Journal of Systems and Software, vol. 164, p. 110542, 2020.

[11] IEC61078, Reliability Block Diagram Method, IEC Standard No. 61078, 1991.

[12] IEC61025, Fault Tree Analysis. IEC Standard No. 61025, 2nd edn., 2006.

[13] E. Ruijters and M. Stoelinga, Fault tree analysis: A survey of the state-ofthe-art in modeling, analysis and tools, Computer science review, vol. 15, pp. 29-62, 2015.

[14] D. Sculley, et al., Hidden technical debt in machine learning systems, In Advances in neural information processing systems, pp. 2503-2511, 2015.

[15] A. Dwarakanath, M. Ahuja, S. Sikand, R. M. Rao, R. J. C. Bose, N. Dubash, and S. Podder, Identifying implementation bugs in machine learning based image classifiers using metamorphic testing, in Proc. of the 27th ACM SIGSOFT International Symposium on Software Testing and Analysis, pp. 118-128 2018.

[16] A. Dwarakanath, M. Ahuja, S. Podder, S. Vinu, A. Naskar, and M. Koushik, Metamorphic testing of a deep learning based forecaster, In Proc. of 2019 IEEE/ACM 4th International Workshop on Metamorphic Testing (MET), pp. 40-47, 2019.

[17] Y. Wang, W. Wang, Y. Cai, B. Hooi, B. C. Ooi, Detecting implementation bugs in graph convolutional network based node classifiers, In Proc. of the 31st International Symposium on Software Reliability Engineering (ISSRE 2020), 2020.

[18] S. Latifi, B. Zamirai, and S. Mahlke, Polygraphmr: Enhancing the reliability and dependability of cnns, In Proc. of 50th IEEE/IFIP International Conference on Dependable Systems and Networks (DSN), pp. 99-112, 2020.

[19] E. Barr, M. Harman, P. McMinn, M. Shahbaz, and S. Yoo, The oracle problem in software testing: A survey, IEEE transactions on software engineering, vol. 41, no. 5, pp. 507-525, 2015.

[20] E. J. Weyuker, On testing non-testable programs, Computer Journal, vol. 25 , no.4, pp.465-470, 1982

[21] C. Murphy, G. E. Kaiser, and M. Arias, An approach to software testing of machine learning applications. In SEKE, vol. 167, 2007.

[22] M. D. Davis and E. J. Weyuker, Pseudo-oracles for non-testable programs, in Proc. of the ACM'81 Conference, pp. 254-257, 1981.

[23] J. M. Zhang, M. Harman, L. Ma, and Y. Liu, Machine learning testing: Survey, landscapes and horizons, IEEE Transactions on Software Engineering, 2020.

[24] W. Wu, H. Xu, S. Zhong, M. Lyu, and I. King. Deep validation: Toward detecting real-world corner cases for deep neural networks. In Proc. of the 49th IEEE/IFIP International Conference on Dependable Systems and 
Networks (DSN), pp. 125-137, 2019.

[25] T. Dreossi, A. Donzé, and S. A. Seshia, Compositional falsification of cyber-physical systems with machine learning components, In NASA Formal Methods Symposium, pp. 357-372, 2017.

[26] A. Wu, A. H. M. Rubaiyat, C. Anton, and H. Alemzadeh, Model Fusion: weighted $\mathrm{N}$-version programming for resilient autonomous vehicle steering control, In Proc. of IEEE International Symposium on Software Reliability Engineering Workshops, pp. 144-145, 2018.

[27] T. Dietterich, Ensemble methods in machine learning, In Proc. of international workshop on multiple classifier systems, pp. 1-15, 2000.

[28] R. E. Schapire, Y. Freund, P. Bartlett, W. S. Lee, Boosting the margin: A new explanation for the effectiveness of voting methods, The annals of statistics, vol. 26, no. 5, pp. 1651-1686, 1998.

[29] G. Brown, J. Wyatt, R. Harris, and X. Yao, Diversity creation methods: a survey and categorisation, Information Fusion, vol. 6, no. 1, pp.5-20, 2015.

[30] D. Partridge, W. Krzanowski, Software diversity: practical statistics for its measurement and exploitation, Information and software technology, vol. 39, no. 10, pp.707-717, 1997.

[31] G. Brown, An information theoretic perspective on multiple classifier systems, In International Workshop on Multiple Classifier Systems, pp. 344-353, 2009.

[32] E. K. Tang, P. N. Suganthan, and X. Yao, An analysis of diversity measures, Machine learning, vol. 65, no.1, pp. 247-271, 2006.

[33] Z.-H. Zhou, Ensemble methods: foundations and algorithms, CRC press, 2012.

[34] Z. Gong, P. Zhong, and W. Hu, Diversity in machine learning, IEEE Access, vol. 7, pp. 64323-64350, 2019.

[35] A. P. Dawid, Conditional independence in statistical theory, Journal of the Royal Statistical Society. Series B (Methodological), vol. 41, no. 1, pp. 1-31, 1979.

[36] W. Xu, D. Evans, and Y. Qi, Feature Squeezing: Detecting adversarial examples in deep neural networks, 25th Annual Network and Distributed System Security Symposium, 2018.

[37] D. E. Eckhardt, L. D. Lee, A theoretical basis for the analysis of multiversion software subject to coincident errors, IEEE Trans. Software Eng., Vol. SE-11, No. 12, pp. 1511-1517, 1985.

[38] B. Littlewood, D.R. Miller, Conceptual modeling of coincident failures in multiversion software, IEEE Trans. on Software Eng., Vol. 15, No. 12, pp.1596-1614, 1989.

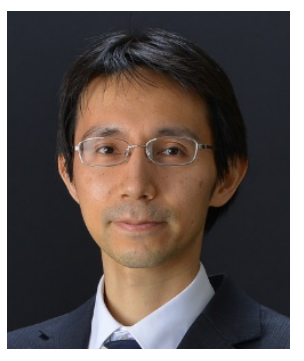

Fumio Machida is an associate professor at the Computer Science Department in University of Tsukuba. Before the current position, he was a principal researcher at NEC Corporation. He was a visiting scholar in the Department of Electrical and Computer Engineering at Duke University in 2010. He received the $\mathrm{PhD}$ degree from Tokyo Institute of Technology in 2018. He was a recipient of the young scientists' prize of Japan in 2014. His research interests include modeling and analysis of system dependability, software aging and rejuvenation, and cloud and edge computing systems. He is a senior member of the IEEE and the member of ACM. 